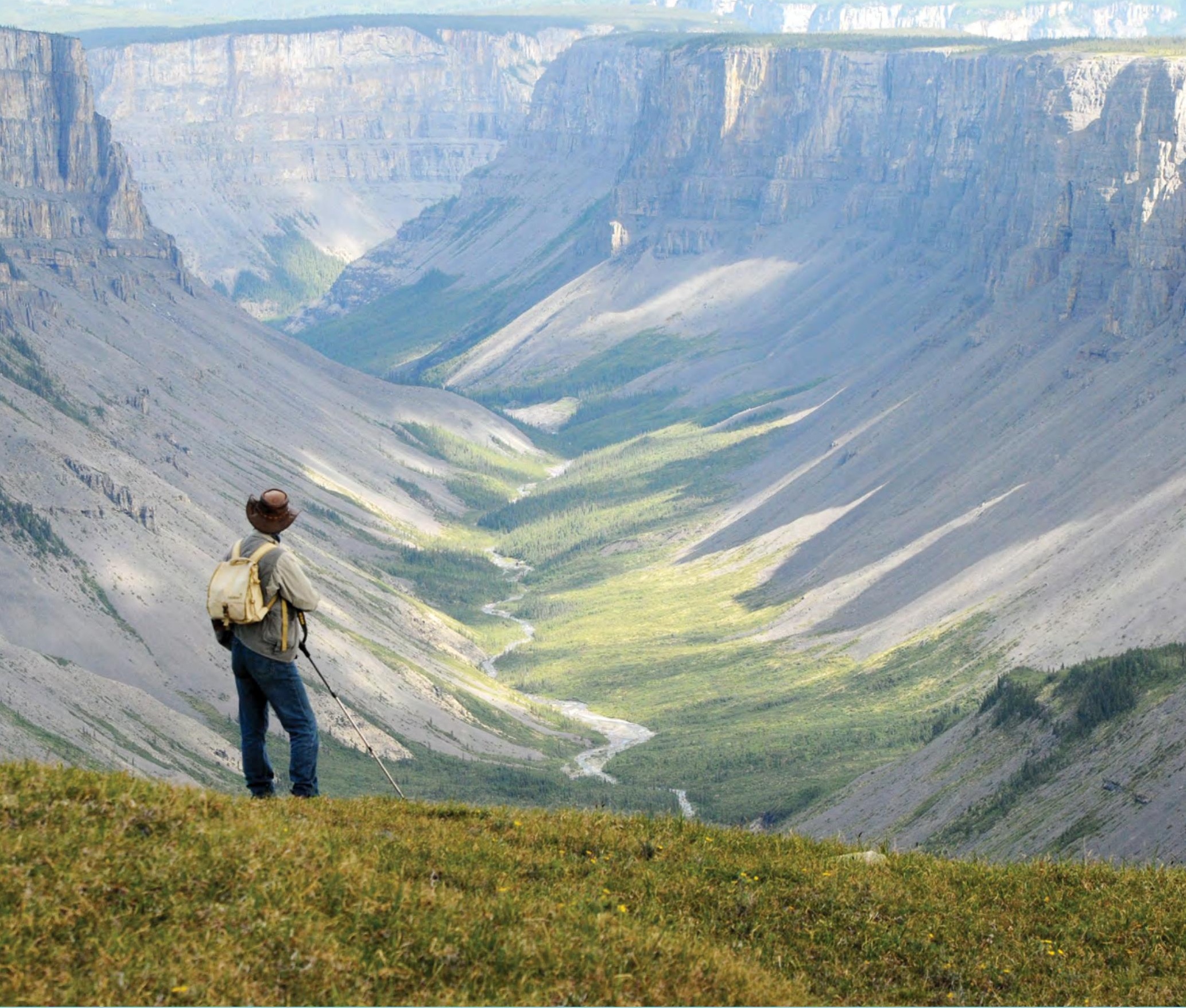

\title{
CHAPITRE 21
}

\section{GESTION DES AIRES PROTIÉGÉES POUR LA DIVERSITÉ BIOLOGIQUE ET LES FONCTIONS ÉCOSYSTIEMIQUES}

\section{Auteurs principaux :}

Stephen Woodley, Kathy MacKinnon, Stephen McCanny, Richard Pither, Kent Prior, Nick Salafsky et David Lindenmayer

\section{TABLE DES MATIÈRES}

- Introduction

- Relation entre biodiversité et fonction écologique

- Évaluation des conditions des aires protégées : intégrité écologique

- Gestion des aires protégées pour la biodiversité

- Gestion des menaces pesant sur les aires protégées

- Suivi et évaluation des conditions écologiques des aires protégées

- Conclusion

- Références 


\section{AUTEURS PRINCIPAUX}

STEPHEN WOODLEY est écologiste et travaille pour la Commission mondiale des aires protégées (CMAP) de l'Union internationale pour la conservation de la nature (UICN), Canada.

KATHY MACKINNON est vice-présidente de la CMAP-UICN, Royaume-Uni.

STEPHEN MCCANNY dirige le groupe de suivi et d'information écologique de Parcs Canada.

RICHARD PITHER est spécialiste de la conservation des espèces à Parcs Canada.

KENT PRIOR est responsable national de la gestion active et de la restauration écologique de Parcs Canada.

NICK SALAFSKY est co-directeur de Foundations of Success, États-Unis.

DAVID LINDENMAYER est professeur d'écologie et lauréat du Conseil de recherche australien à la Fenner School of Environment and Society de l'Université nationale australienne.

\section{REMERCIEMENTS}

Nous remercions lan Pulsford et trois relecteurs anonymes pour leurs commentaires utiles sur le manuscrit. Nous remercions James MacKinnon pour sa contribution.

\section{CITATION}

Woodley, S., MacKinnon, K., McCanny, S., Pither, R., Prior, K. Salafsky, N. et Lindenmayer, D. Managing protected areas for biological diversity and ecosystem functions. In Worboys, G. L., Lockwood, M., Kothari, A., Feary, S. et Pulsford, I. (éd.) Gouvernance et gestion des aires protégées. Canberra : ANU Press, 2020. doi.org/ 10.22459/GGAP.21

\section{PHOTO DE LA PAGE DE TITRE}

Le plateau de Nahanni Ram, dans le parc national Nahanni, Territoires du Nord-Ouest, Canada

Source : Alison Woodley 


\section{Introduction}

Les aires protégées sont la pierre angulaire des efforts mondiaux de conservation de la biodiversité. La diversité biologique (biodiversité) et les fonctions écosystémiques sont les composantes fondamentales de tout écosystème (encadré 21.1) que les gestionnaires d'aires protégées doivent prendre en compte pour atteindre leur but. Ce chapitre examine la relation entre diversité biologique et fonctions écologiques, les menaces qui pèsent sur chacune d'elles et la façon d'évaluer et de réaliser un suivi des écosystèmes.

De plus en plus, les aires protégées représentent les derniers endroits disponibles pour une grande partie de la biodiversité de la planète. La Liste rouge de l'Union internationale pour la conservation de la nature (UICN) des espèces menacées, présentant un risque élevé d'extinction mondiale, révèle que beaucoup de ces espèces ne sont présentes aujourd'hui que dans des aires protégées (le Saout et al., 2013). Par exemple, le rhinocéros de Java (Rhinocéros sondaicus) n'est plus présent que dans le parc national d'Ujung Kulon, en Indonésie. De même, le grand rhinocéros indien (Rhinoceros unicornis), autrefois répandu dans toute l'Asie, est aujourd'hui limité aux aires protégées, dont le parc national de Kaziranga, en Inde, et le parc national Royal Chitwan, au Népal. Conserver la biodiversité dans les aires protégées signifie conserver à la fois les espèces et les fonctions écologiques dont dépendent ces espèces.

\section{Relation entre biodiversité et fonction écologique}

La nécessité de gérer la diversité biologique, y compris les fonctions écologiques, est inhérente à la définition de l'UICN d'une aire protégée. Une aire protégée est « un espace géographique clairement défini, reconnu, consacré et géré, par des moyens juridiques et autres moyens efficaces, pour parvenir à la conservation à long terme de la nature et des services écosystémiques et valeurs culturelles associés " (Dudley, 2008:8).

Du point de vue de la conservation, un rôle clé des aires protégées est de maintenir les structures écologiques (gènes, espèces et écosystèmes) et les fonctions écologiques qui les soutiennent. Outre la protection de la biodiversité, les aires protégées jouent également un rôle clé dans la protection des services écosystémiques, qui sous-tendent le bien-être humain (voir chapitre 6). Les aires protégées conservent également les éléments non vivants ou abiotiques des écosystèmes. Par exemple, les aires protégées préservent la diversité géologique, ainsi que la biodiversité associée à certaines caractéristiques géologiques (chapitre 18).

\section{Encadré 21.1 Définitions clés}

La diversité biologique, ou biodiversité pour faire court, désigne la variabilité entre les organismes vivants de toutes origines, y compris les écosystèmes terrestres, marins et autres écosystèmes aquatiques, et les complexes écologiques dont ils font partie. Cela inclut la diversité au sein des espèces, entre les espèces et la diversité des écosystèmes. La biodiversité est mesurée à trois niveaux hiérarchiques principaux :

- La diversité génétique comprend les différents gènes contenus dans toute plante, animal, champignon ou micro-organisme individuel. La diversité des espèces est une mesure du nombre d'espèces et de la régularité de leur répartition au sein d'une communauté écologique. La diversité des écosystèmes comprend les différents habitats, communautés biologiques et processus écologiques, ainsi que les variations au sein des écosystèmes individuels.

- Les fonctions écosystémiques sont les processus physiques, chimiques et biologiques qui contribuent au fonctionnement d'un écosystème. Certaines définitions de la biodiversité incluent les fonctions écosystémiques. La productivité primaire (conversion de la lumière du soleil en énergie), le cycle des nutriments et le cycle de l'eau sont des exemples de fonctions écosystémiques.

- Les services écosystémiques sont les avantages que les écosystèmes apportent à l'humanité, y compris des produits tels que les aliments et l'eau potable, et des processus tels que la pollinisation, la décomposition des déchets ou la régulation des inondations. Les services écosystémiques forment un sous-ensemble de la diversité biologique et des fonctions écosystémiques, d'un point de vue humain (Daily, 1997).

L'interaction de la structure écologique, principalement les espèces, avec les fonctions écologiques est complexe et demeure mal comprise. Des questions clés demeurent, telles que, certaines espèces sont-elles redondantes ? Si une espèce disparaît d'un écosystème (ou d'une aire protégée), cela affectera-t-il les fonctions écologiques à cet endroit ? La compréhension de cette relation devient de plus en plus critique avec le rythme accru auquel les espèces sont éliminées des écosystèmes par des extirpations locales (disparition des espèces d'une zone donnée), voire des extinctions (disparition complète des espèces) (voir Butchart et al., 2010).

Les préoccupations concernant la relation entre structure et fonction écologiques ont donné lieu à une série d'études scientifiques, telles que l'Évaluation des écosystèmes pour le millénaire, commandée par des organismes internationaux (Schulze et Mooney, 1993; Heywood, 1995), ou les travaux 


\section{Encadré 21.2 Six déclarations de consensus sur la relation entre structure (espèces) et fonction écologiques}

1. II existe aujourd'hui des preuves sans équivoque que la perte de biodiversité réduit l'efficacité avec laquelle les communautés écologiques fonctionnent, y compris la production de biomasse, et la décomposition et le recyclage de nutriments biologiquement essentiels (figure 21.1).

2. De plus en plus de preuves indiquent que la biodiversité augmente la stabilité des fonctions écosystémiques au fil du temps.

3. L'impact de la biodiversité sur un processus écosystémique donné n'est pas linéaire. La plupart des études expérimentales indiquent que les pertes initiales de biodiversité dans divers écosystèmes ont des impacts relativement faibles sur les fonctions écosystémiques, mais que des pertes croissantes entraînent une accélération des taux de changement (figure 21.1).

4. Les communautés biologiquement diverses sont plus productives parce qu'elles contiennent des espèces clés ayant une grande influence sur la productivité. Les différences de caractères fonctionnels entre les organismes augmentent la capture totale des ressources. Les fonctions écosystémiques sont donc contrôlées à la fois par l'identité et la diversité des organismes.

5. Une perte de diversité à tous les niveaux trophiques a le potentiel d'influencer les fonctions écosystémiques encore plus fortement qu'une perte de diversité à l'intérieur des niveaux trophiques. Les interactions à l'intérieur des réseaux trophiques sont des médiateurs clés du fonctionnement des écosystèmes. La perte de consommateurs de rang supérieur peut produire un effet de cascade sur l'ensemble d'un réseau trophique, entraînant des altérations de la structure de la végétation, de la fréquence des incendies et même des épidémies dans divers écosystèmes.

6. Les caractéristiques fonctionnelles des organismes ont des impacts importants sur l'ampleur des fonctions écosystémiques, de sorte que la perte d'une espèce spécifique peut avoir des impacts très variables sur la fonction de l'écosystème. La mesure dans laquelle les fonctions écologiques changeront après l'extinction d'une espèce particulière dépendra grandement des traits biologiques ayant disparu.

Source : Adapté de Cardinale et al. (2012)

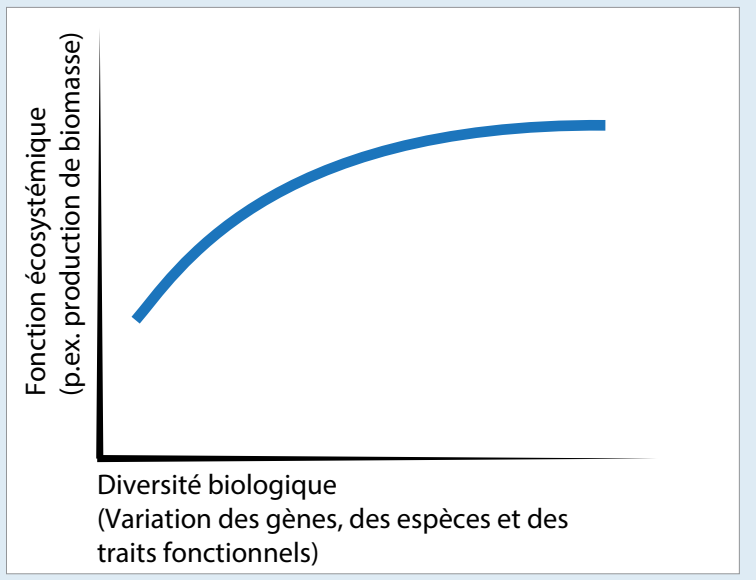

Figure 21.1 Relation entre les fonctions écologiques et la biodiversité

Source : Cardinale et al. (2012)

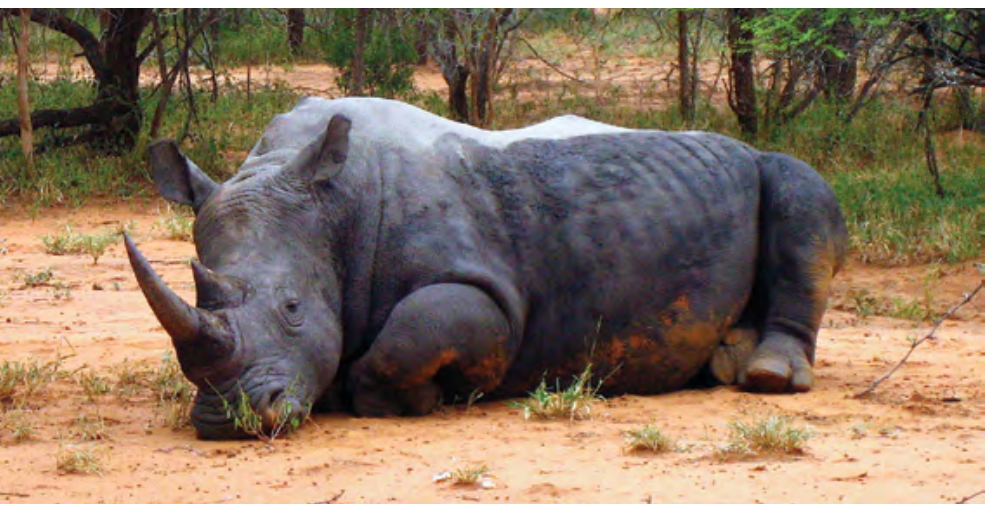

Le rhinocéros blanc (Ceratotherium simum) et autres grands herbivores jouent un rôle important dans le fonctionnement des écosystèmes en Afrique, et nécessitent de vastes zones d'habitats sécurisées, Réserve de faune sauvage de Mkhuze, Afrique du Sud

Source : lan Pulsford du World Resources Institute (2005). Un important document de synthèse publié dans Nature (Cardinale et al., 2012) a résumé la littérature mondiale disponible sur la relation entre structure et fonction écologiques au moyen de six déclarations de consensus (encadré 21.2).

Les leçons à tirer de ces considérations pour les gestionnaires d'aires protégées incluent :

- Les aires protégées devraient être gérées, dans la mesure du possible, de manière à conserver toutes les espèces autochtones, afin de maintenir leur fonction écologique et, en fin de compte, leur intégrité écologique. La meilleure politique est de supposer que toutes les espèces sont importantes.

- La gestion des aires protégées devrait se concentrer sur l'identification et le maintien des processus écologiques reconnus comme importants pour 


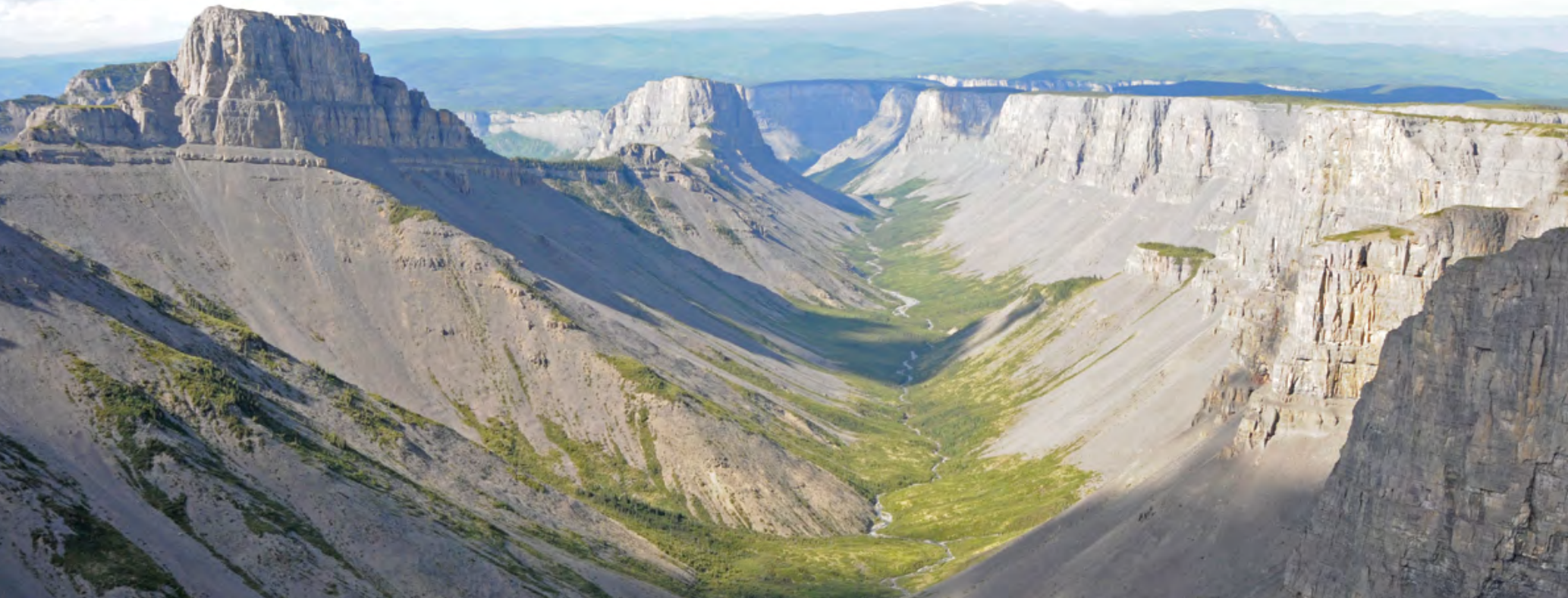

Le parc national de Nahanni, au Canada, contient certaines des rivières sauvages les plus spectaculaires d'Amérique du Nord, avec des canyons profonds, d'énormes cascades et des terrains karstiques spectaculaires, des systèmes de grottes et des sources chaudes

Source : Alison Woodley

l'écosystème en question. Cela inclut les processus perturbateurs tels que le feu (pour les écosystèmes adaptés au feu) et les inondations.

- Les gestionnaires devraient accorder une attention particulière au maintien des rôles fonctionnels des espèces à différents niveaux trophiques (niveaux dans un réseau alimentaire). Par exemple, il est bien entendu que les grands prédateurs assurent une régulation descendante des écosystèmes et sont essentiels au maintien de l'intégrité écologique (étude de cas 21.6).

\section{Schémas de la biodiversité mondiale}

La diversité de la vie dans une aire protégée est déterminée par les mêmes facteurs qui déterminent les schémas de la vie sur Terre. D'une manière générale, ces schémas sont clairs et faciles à décrire. Lorsqu'il s'agit de la biodiversité d'une aire protégée individuelle, cependant, les schémas mondiaux à grande échelle sont fortement influencés par des facteurs locaux. Pour une analyse détaillée des schémas de la diversité mondiale, on pourra se reporter à Gaston (2000) (voir aussi le chapitre 3). La plupart des analyses de variation spatiale concernent la biodiversité telle que mesurée par le nombre d'espèces observées ou estimées dans une zone (richesse spécifique). Une plus grande attention a été accordée à la variation latitudinale de la richesse spécifique, et on en sait relativement peu sur la variation de la diversité des gènes, des individus ou des populations le long de gradients latitudinaux.

Les schémas généraux de la biodiversité sont les suivants :
- En général, la majorité des espèces terrestres et d'eau douce se trouvent sous les tropiques, la richesse spécifique diminuant progressivement des tropiques vers les pôles. Ce schéma général est également valable pour les océans.

- En général, on trouve des niveaux plus élevés de biodiversité à basse altitude, dans les zones où les précipitations annuelles sont plus élevées, et dans les zones où les températures estivales sont plus chaudes.

- En général, différents taxons (catégories d'espèces, comme les reptiles) présentent le même type de variation régionale. Par exemple, à l'échelle mondiale, des zones de grande diversité d'oiseaux présenteront également une grande diversité de plantes ou d'amphibiens (voir la figure 21.2). Cependant, ce schéma général présente d'énormes variations. $\mathrm{Au}$ niveau régional, il est impossible de prédire raisonnablement la diversité d'un taxon uniquement à partir de la diversité d'un autre taxon.

- Ces schémas généraux sont importants à grande échelle, mais n'expliquent pas toujours les conditions locales dans les aires protégées. La biodiversité peut être fortement modifiée par le terrain, la pente, l'eau, le substrat rocheux, le type et le développement du sol, ou l'histoire de la colonisation écologique et des perturbations.

Il est important de prendre en compte à la fois la biogéographie régional et locale dans la gestion d'une aire protégée (encadré 21.3). De nombreux efforts ont été déployés pour décrire systématiquement les schémas globaux d'organisation biologique (Klijn et de Haes, 1994). De nombreuses listes et schémas de classification écologique des terres ont été élaborés à partir des exemples suivants : 


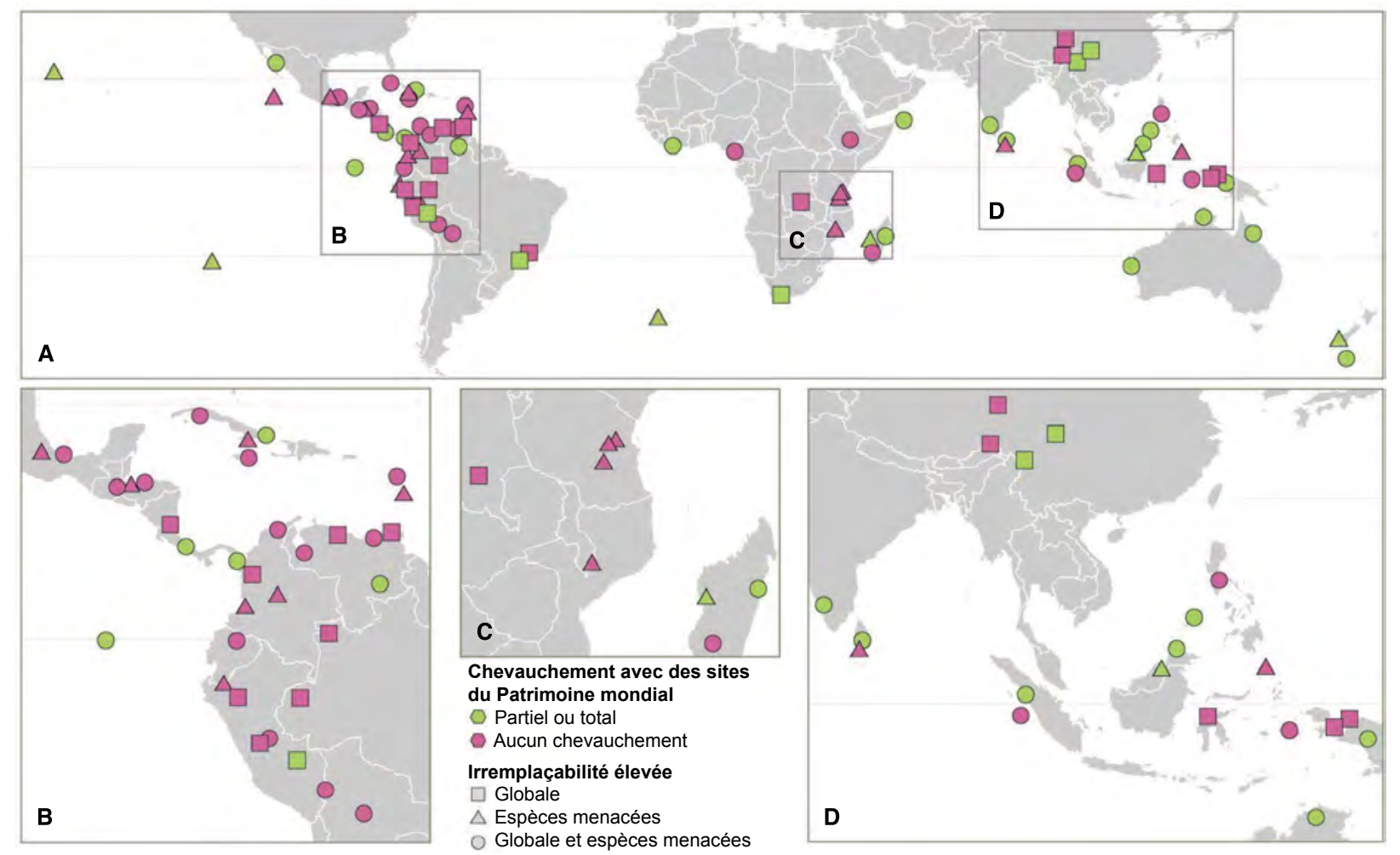

Figure 21.2 Certaines des aires protégées les plus importantes au monde pour la conservation des espèces d'amphibiens, d'oiseaux et de mammifères : (A) répartition mondiale, (B) Amérique centrale et Amérique du Sud, (C) Afrique de l'Est, (D) Asie du Sud et du Sud-Est

Source : Adapté de Le Saout et al. (2013)

\section{Encadré 21.3 Un système imbriqué simple de classification des écosystèmes et de leurs utilisations pour la gestion des aires protégées}

Biome : une grande communauté naturelle de flore et faune occupant un habitat majeur, par exemple, la forêt ou le désert.

Écorégion : un modèle d'écosystèmes associés à des combinaisons caractéristiques de sol, à des formes de relief et à une végétation à l'échelle régionale, qui caractérisent cette région, par exemple, les forêts d'acacia-Miombo.

Écodistrict : subdivision d'une écorégion dont les sols, la topographie et la végétation sont plus uniformes, par exemple, les collines boisées d'acacia exposées au sud.

Source : Klijn (1994)

- Biogéographie : la classification écologique des terres est un ensemble d'approches organisant les types d'écosystèmes mondiaux en fonction du climat, de la topographie et de la végétation. Certaines approches, comme les " provinces biogéographiques ", tiennent compte à la fois de la flore et de la faune.
- Botanique : les botanistes ont identifié des «provinces floristiques" basées sur des communautés florales.

- Zoologie : les zoologistes ont identifié des « provinces zoogéographiques " basées sur des communautés fauniques, et ont même identifié des systèmes basés sur des groupes, tels que les "provinces de mammifères ».

- Géologie et pédologie (étude des sols) : la matière physique et l'énergie qui constituent la Terre.

Le plus utile pour la gestion des aires protégées est peutêtre le "système de classification écologique des terres et des mers ", qui intègre une gamme de facteurs écologiques, plutôt que de se concentrer sur un seul élément. Les unités écologiques peuvent être utilement décrites sur la base du substrat rocheux, du climat, de la physiographie et de la végétation correspondante, créant ainsi un système de classification écologique des terres.

Les références classiques sont la carte et le document de l'UICN préparé par Udvardy (1975) et intitulée : Une classification des provinces biogéographiques du monde. Du point de vue des aires protégées, le Fonds mondial pour la nature (WWF) a dirigé le développement de systèmes mondiaux de classification écologique, comprenant des cartes et des descriptions en ligne. Les lecteurs sont 
également invités à consulter les descriptions globales des écorégions terrestres élaborées par Olson et al. (2001), pour la meilleure description actuelle des écorégions terrestres. Pour les régions marines côtières, les lecteurs sont invités à se référer à Spalding et al. (2007).

Ces systèmes de classification sont essentiels pour de nombreux aspects de la gestion des aires protégées tels que :

- planification régionale de la conservation visant à évaluer les lacunes du système d'aires protégées ;

- fixer des objectifs pour la représentation des aires protégées et la planification de la conservation ;

- déterminer le niveau d'importance régionale ou mondiale d'une aire protégée ;

- évaluer les conditions de caractéristiques écologiques (par exemple, cartographie des types d'écosystèmes, bassin versant intact) ;

- rapport sur les conditions des aires protégées;

- étudier les régimes de perturbation naturelle dans un contexte plus vaste qu'une aire protégée ;

- définir des zones semencières pour les projets de restauration.

La répartition spatiale des espèces menacées peut être obtenue en examinant où le système actuel d'aires protégées contient des espèces figurant sur la Liste rouge de l'UICN (figure 21.2).

Il est important de penser aux aires protégées et aux réseaux d'aires protégées dans le contexte des modèles mondiaux de biodiversité. Cependant, ce n'est pas parce qu'une aire protégée n'est pas située dans une zone à forte biodiversité que sa valeur pour la conservation de la nature sera moindre. Une conservation efficace exige une représentation adéquate de toutes les espèces et de tous les écosystèmes dans les aires protégées (Woodley et al., 2012). En outre, certaines espèces importantes, préoccupantes pour la conservation, se trouvent dans des endroits pauvres en espèces, comme les plantes vivant dans des environnements très riches en minéraux.

\section{Évaluation des conditions des aires protégées : intégrité écologique}

Par définition, les aires protégées sont établies pour préserver la nature, et l'écosystème doit être en bon état pour conserver sa biodiversité à long terme. La gestion d'une aire protégée dépend de la connaissance des conditions écologiques de cette aire protégée en tant qu'élément fondamental de la gestion. Sur la base de cette information, entre autres, des décisions peuvent être prises concernant la gestion et la restauration. Les tâches clés de la gestion des aires protégées sont : 1) comprendre le fonctionnement de l'écosystème, 2) définir des indicateurs appropriés pour évaluer les conditions écologiques, 3) réaliser un suivi de ces indicateurs et déterminer les conditions écologiques, et 4) mettre en ouvre des mesures de gestion lorsque ces indicateurs dépassent une fourchette acceptable. Le suivi des informations sur les conditions écologiques et les résultats des mesures de gestion est au cœur du processus de gestion (figure 21.3).

\section{Gestion de l'intégrité écologique}

Historiquement, les aires protégées ont été gérées avec des objectifs imprécis, comme la conservation des zones " naturelles " ou " sauvages ». Dans la pratique, de nombreuses aires protégées ont été gérées avec des objectifs spécifiques de conservation des espèces à l'esprit, comme le maintien de grands troupeaux de gibier dans les parcs

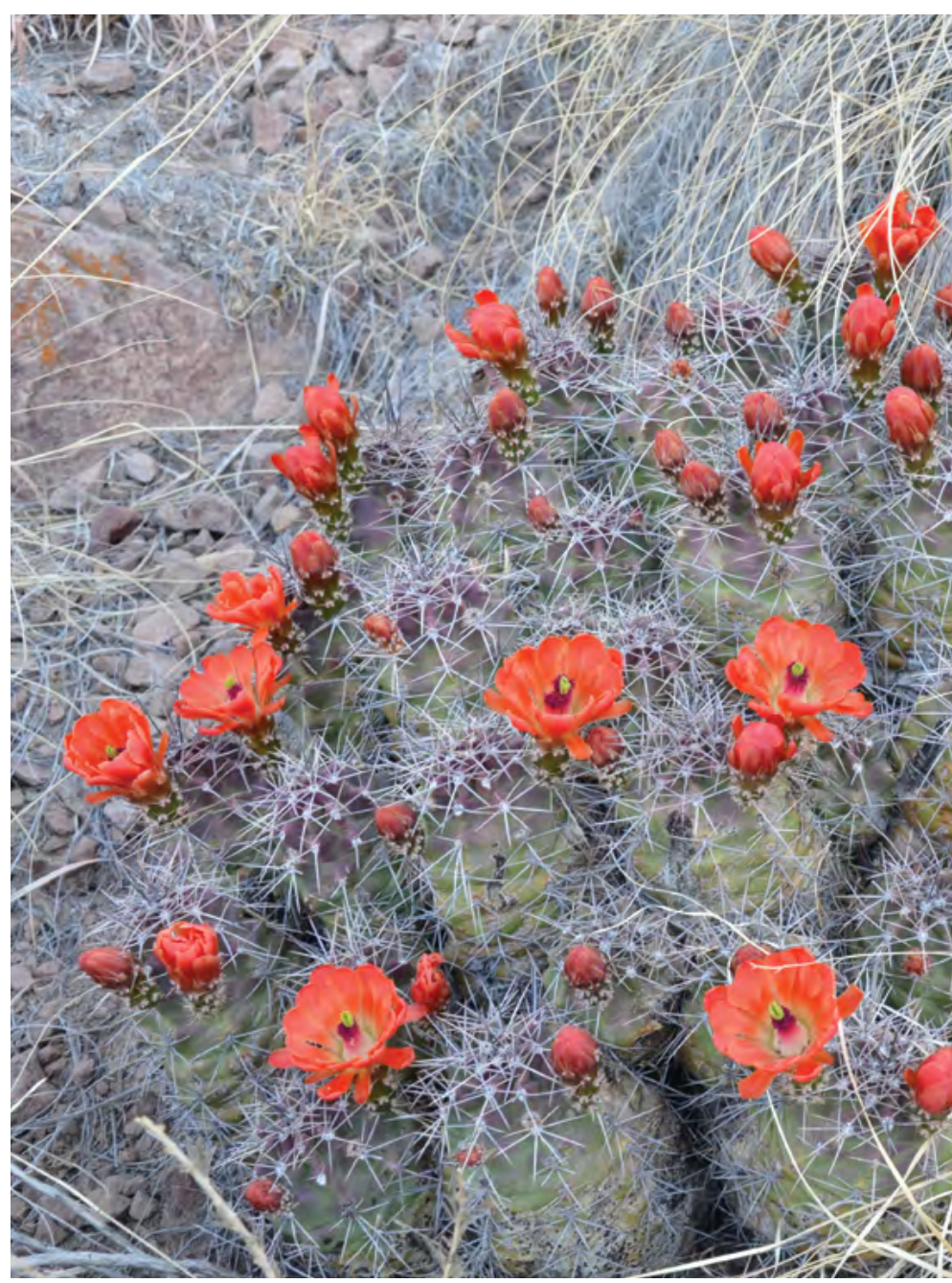

Cactus Opuntia (Opuntia sp.), parc national de Big Bend, Texas, États-Unis

Source : Stephen Woodley 


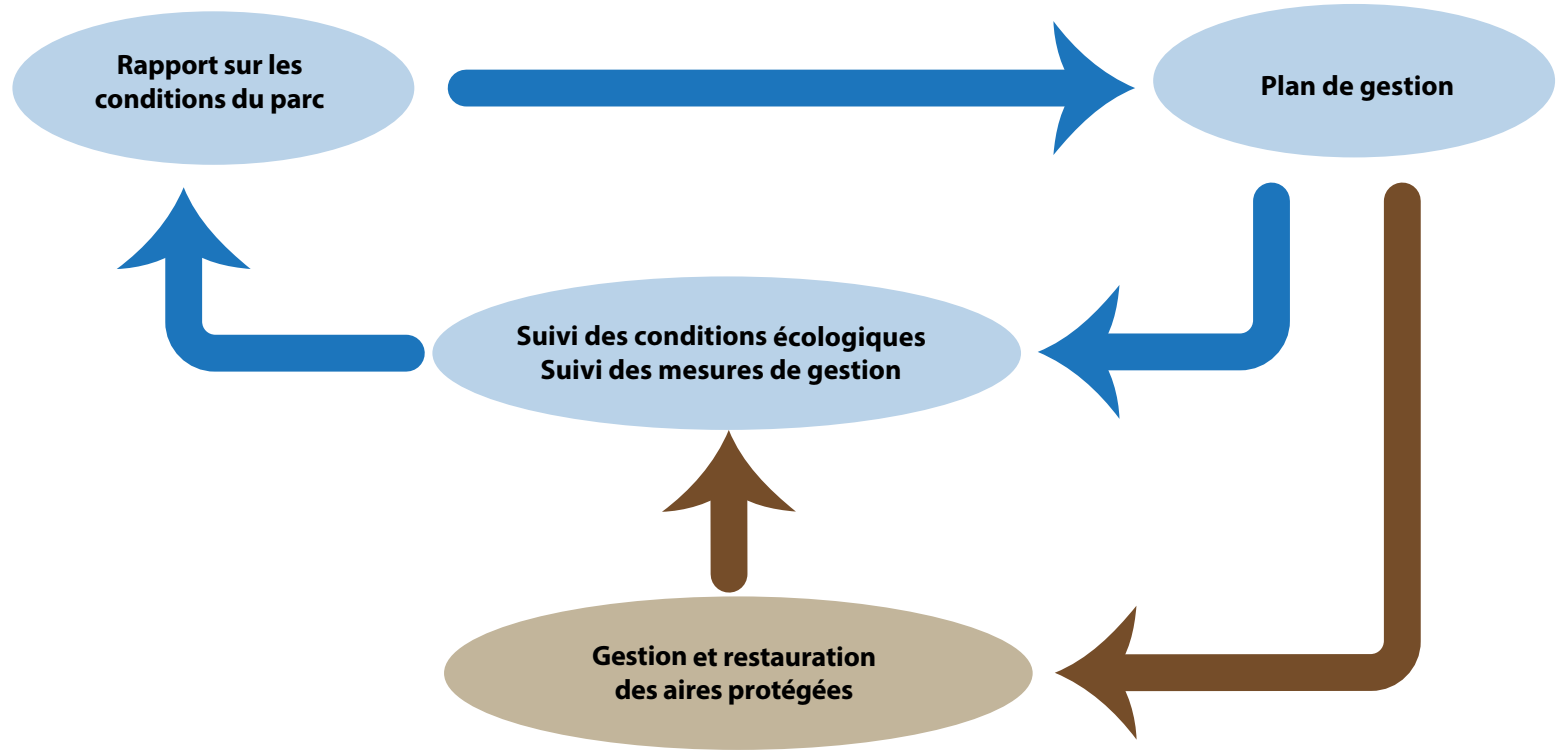

Figure 21.3 Boucle de gestion des aires protégées

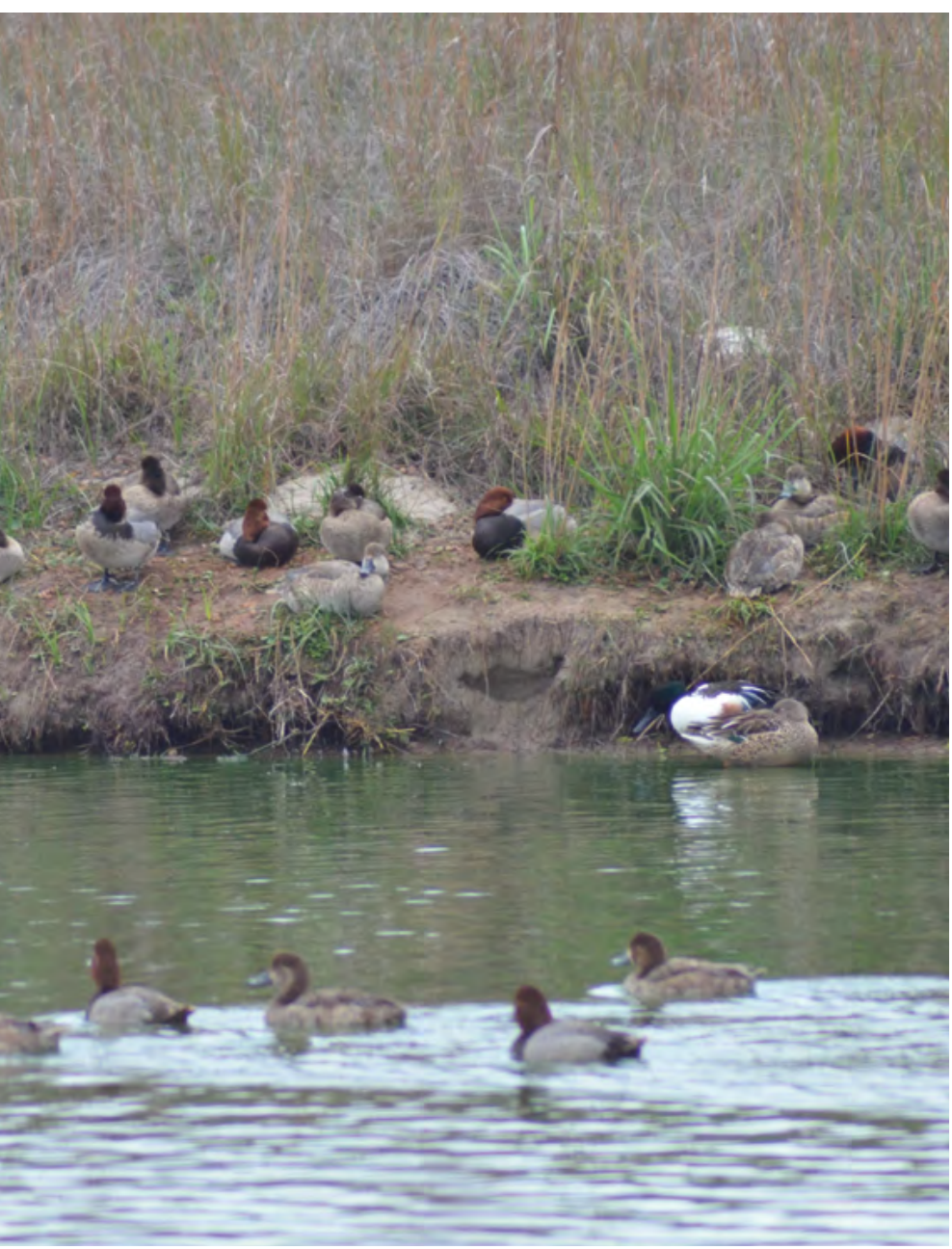

Des oiseaux d'eau au repos sur le littoral national de l'île Padre, Texas, États-Unis, lors de leur migration annuelle

Source : Stephen Woodley africains, ou d'espèces phares telles que les tigres et les éléphants dans les grands parcs indiens (MacKinnon et al., 1986). Les gestionnaires d'aires protégées adoptent de plus en plus les termes "intégrité écologique " et "santé des écosystèmes " pour décrire leurs objectifs en matière de gestion des écosystèmes. De nombreuses lois et déclarations politiques officielles articulent aujourd'hui le concept d'intégrité comme un objectif, y compris l'Accord sur la qualité de l'eau dans les Grands Lacs (International Joint Commission, 1978) et la Convention sur la diversité biologique (CBD, 2004). La notion d'intégrité écologique a été discutée sous plusieurs angles (Edwards et Regier, 1990 ; Woodley et al., 1993 ; Pimentel et al., 2000). En ce qui concerne une aire protégée, l'intégrité écologique désigne une condition caractéristique de sa région naturelle et susceptible de persister, y compris les composantes abiotiques et la composition et l'abondance des espèces et des communautés biologiques autochtones, les taux de changement et les processus de soutien. Notez que cette définition est basée sur une perspective écologique et qu'elle ne n'implique pas l'absence de personnes. En fait, l'intégrité écologique est un concept susceptible de s'appliquer aux écosystèmes où des personnes sont présentes ou non, et où ces personnes jouent un rôle écologique clé ou non.

Les leçons à tirer des meilleures pratiques visant à déterminer les conditions écologiques d'une aire protégée incluent :

- Toutes les aires protégées doivent disposer de buts et d'objectifs de gestion clairs en ce qui concerne la biodiversité et les processus écosystémiques. Dans le cas contraire, l'objectif de gestion est imprécis.

- Les objectifs écologiques devraient être inclus dans le plan de gestion de l'aire protégée. 
- Envisager d'utiliser l'intégrité écologique comme objectif final de gestion. Elle a été adoptée par de nombreux organismes chargés des aires protégées et de nombreuses informations sont disponibles sur la façon de la mesurer.

\section{Intégrité écologique et aires protégées}

Comprendre l'intégrité écologique dans le contexte des aires protégées nécessite une réflexion approfondie sur la structure et le fonctionnement d'un écosystème. Grâce à la science de l'écologie, nous comprenons que les écosystèmes présentent un certain nombre de caractéristiques qu'il est important de mesurer (Woodley, 2010).

1. Les aires protégées devraient conserver toutes les espèces autochtones. Les écosystèmes perdent leur intégrité lorsqu'ils perdent des espèces. Parmi les principales causes de perte d'espèces se trouvent la perte et la fragmentation des habitats. De nombreuses aires protégées perdent des espèces parce qu'elles sont trop petites. Par exemple, les parcs de l'ouest de l'Amérique du Nord ont connu des taux d'extinction inversement liés à la taille du parc (Newmark, 1995). Parmi les autres exemples d'écosystèmes stressés, perdant des espèces, mentionnons les forêts boréales canadiennes, sujettes à de fortes émissions de dioxyde de soufre (Freedman et Hutchinson, 1980), les forêts décidues tempérées exposées à des radiations (Woodwell, 1970), et les communautés estuariennes de diatomées sujettes à la pollution par les métaux lourds (Patrick, 1967).

2. Les populations d'espèces dans les aires protégées doivent être viables. Pour des raisons pratiques, il sera possible, pour les gestionnaires d'aires protégées, de vérifier seulement la viabilité de quelques espèces, appelées espèces indicatrices. Il existe de nombreux ouvrages sur la sélection des espèces indicatrices (voir Simberloff, 1998 ; Lindenmayer et Lichens, 2010). La situation des espèces indicatrices est habituellement déterminée en analysant les taux de vitalité des populations (par exemple, les naissances, les décès, l'immigration et l'émigration), et en utilisant ces paramètres pour déterminer la probabilité de survie (ou inversement la probabilité d'extinction), généralement sur 100 ou 1000 ans (Soulé et Simberloff, 1986).

3. Les niveaux trophiques des écosystèmes dans les aires protégées devraient être intacts. Les écosystèmes possèdent des niveaux et des interactions caractéristiques de producteurs primaires, d'herbivores et de carnivores, souvent décrits comme des réseaux trophiques. Les écosystèmes fortement touchés ont tendance à présenter des réseaux trophiques plus simples que les écosystèmes non modifiés. Par exemple, la perte des carnivores supérieurs peut entraîner une hyper-abondance des populations d'ongulés, entraînant des effets néfastes en cascade sur les communautés végétales (Estes et al., 2011 ; voir également études de cas 21.5 et 21.6).

4. Les régimes de perturbation dans les aires protégées devraient être conservés afin de maintenir un mélange de classes d'âge dans les communautés biologiques. Les écosystèmes sont intrinsèquement dynamiques, définis par le feu, le climat, la météo et les herbivores. Après une perturbation, les écosystèmes passent par des stades de succession parfois prévisibles. Les perturbations répétées créent une mosaïque de communautés biologiques dans le temps et dans l'espace. La configuration résultante de types de communautés de taille et d'âge différents détermine la survie des espèces individuelles. Étant donné que certaines perturbations (par exemple, les incendies et les herbivores) peuvent être influencées par les gestionnaires d'aires protégées, cet aspect de l'intégrité écologique devrait, au moins, faire l'objet d'un contrôle de gestion partiel (étude de cas 21.1).

5. La productivité et la décomposition dans les aires protégées devraient être conservées dans les limites de la persistance du système. La plupart des écosystèmes dépendent de la productivité primaire, c'est-à-dire de la quantité de matière organique produite par l'activité biologique par unité de surface au cours d'une période donnée (Hooper et al., 2012). L'apparition de problèmes écosystémiques se fait sentir lorsque des changements subtils de productivité se produisent, et des problèmes majeurs sont signalés lorsque l'énergie de l'écosystème est perdue de manière incontrôlée. Par exemple, dans les systèmes soumis à des contraintes, comme les forêts fortement exploitées, les taux de décomposition augmentent considérablement. La productivité et la décomposition se maintiennent à l'intérieur d'une fourchette pour des écosystèmes spécifiques. Lorsque ces processus vitaux sortent de cette fourchette, l'écosystème est fondamentalement affecté et perd son intégrité. Les changements de productivité peuvent être mesurés à l'aide d'un indice satellite facilement accessible, appelé "indice de végétation par différence normalisée " (NDVI) (Tucker et al., 2005).

6. Le cycle des éléments nutritifs dans les aires protégées devrait se situer dans les limites de la persistance du système. Dans pratiquement tous les écosystèmes, la disponibilité des éléments nutritifs est un facteur limitant, et les taux de cycle des éléments nutritifs sont essentiels au fonctionnement de l'écosystème (Hooper et al., 2012). Les écosystèmes recyclent et 


\section{Étude de cas 21.1 Planification des aires protégées à l'aide d'informations sur les régimes de perturbation, Australie}

De nombreux facteurs sous-tendent la conception des aires protégées, y compris les espèces, les communautés, les écosystèmes ou les processus écologiques ciblés par la conservation. La prise en compte des impacts des perturbations naturelles majeures sur la biodiversité et les principaux processus écologiques est une question importante dans la conception des aires ou des réseaux d'aires protégés. Dans les écosystèmes où les perturbations sont récurrentes et de grande magnitude, ces processus sont essentiels pour des valeurs écologiques importantes, ainsi que pour les populations d'espèces préoccupantes en matière de conservation (Lindenmayer et Franklin, 2002).

Les forêts d'Eucalyptus regnans des hautes terres centrales de l'État du Victoria, dans le Sud-Est de l'Australie, fournissent une illustration précieuse des interrelations entre la conception des aires protégées et les perturbations naturelles. Ces forêts abritent certaines des plantes à fleurs les plus hautes du monde, certains arbres les plus anciens atteignant des hauteurs de 100 mètres. Les forêts d'Eucalyptus regnans offrent un habitat à de nombreuses espèces, y compris le phalanger de Leadbeater (Gymnobelideus leadbeateri), une espèce en voie de disparition à l'échelle mondiale, et centrale de cet écosystème (Lindenmayer, 2009).

Le feu est la principale forme de perturbation naturelle dans les forêts d'Eucalyptus regnans. Avant la colonisation européenne, le régime du feu était peu fréquent, des feux de forêt se produisant en fin d'été (Ashton, 1981) et à une intensité qui permettait à certains arbres de survivre. Les forêts d'Eucalyptus regnans ont été altérées par plus d'un siècle d'exploitation forestière intensive, une augmentation des feux de forêt et la combinaison de ces deux facteurs (Lindenmayer et al. 2011). Environ 20 \% de la forêt d'Eucalyptus regnans des hautes terres centrales du Victoria sont actuellement protégés. Cependant, la taille globale du réseau d'aires protégées est trop petite pour maintenir les forêts et les populations viables d'espèces telles que le phalanger de Leadbeater. Une plus grande superficie de forêt protégée est nécessaire, en particulier si d'autres incendies se produisent au cours des 50 à 100 prochaines années. Tout système de réserve élargi devra être suffisamment grand pour que, même en cas de feu de forêt, il reste suffisamment d'habitat forestier pour soutenir des populations viables d'espèces marsupiales rares (Baker, 1995).

Plusieurs facteurs peuvent être utilisés pour déterminer où une zone étendue de forêt réservée pourrait être mieux située. L'expansion devrait inclure des endroits reliant à la fois des zones clés de l'habitat d'espèces cibles, telles que le phalanger de Leadbeater, et les réserves existantes. Une connectivité écologique accrue permettra la dispersion des espèces dans les paysages forestiers, y compris ceux en phase de régénération après un feu de forêt. Deuxièmement, une zone élargie de forêt réservée devra englober des zones de forêt ancienne, ainsi que des zones susceptibles de fournir un habitat convenable pour les espèces cibles, comme le phalanger de Leadbeater (Lindenmayer et al., 1999).

Cette étude de cas souligne l'importance d'intégrer les effets des perturbations dans la conception et l'établissement d'aires protégées efficaces.

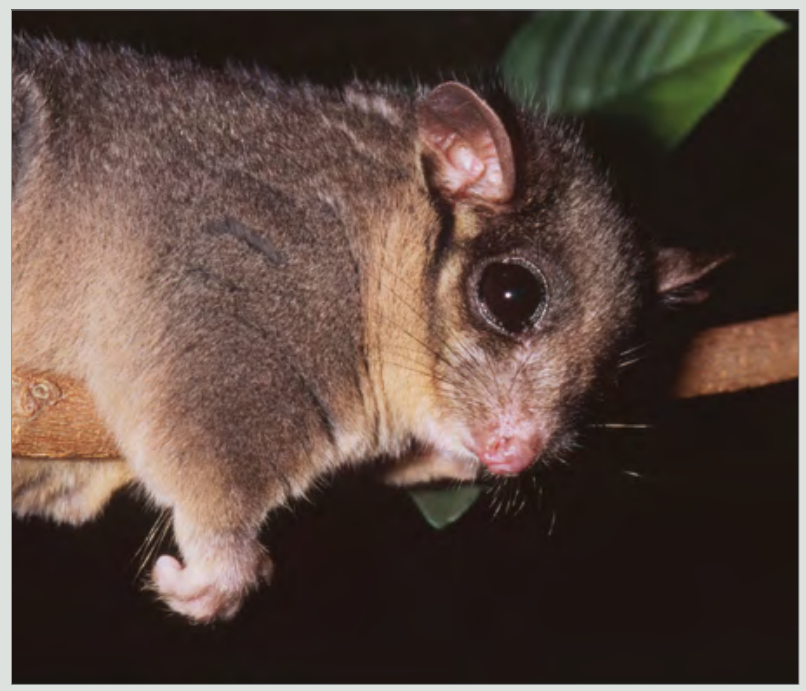

Phalanger de Leadbeater (Gymnobelideus leadbeateri), en voie de disparition, Victoria, Australie

Source : David Lindenmayer conservent les nutriments à des taux caractéristiques. Au fur et à mesure que le niveau de stress des écosystèmes augmente et que ceux-ci perdent leur intégrité, ils perdent leur capacité à retenir les éléments nutritifs et présentent des changements dans les taux de cycle des éléments nutritifs et dans l'abondance relative des sources de nutriments (Likens et al., 1978). Les gammes de cycles des éléments nutritifs peuvent être déterminées à partir des valeurs de la littérature scientifique, et par comparaison avec des écosystèmes de référence sains.
Plus important encore, le concept d'intégrité écologique fournit une base mesurable et claire pour la gestion des aires protégées. Si les buts et objectifs des aires protégées ne sont pas mesurables, il n'y a aucun moyen de savoir si la gestion est efficace ou non (Lindenmayer et Lichens, 2010). Ceci est particulièrement important lorsque une gestion et des interventions actives se produisent dans les processus écosystémiques. L'intégrité écologique fournit un cadre permettant de traduire des objectifs généraux de protection de la nature, souvent vagues, en des objectifs plus précis et mesurables, fondés sur des conditions écologiques souhaitables, pouvant être suivies. 


\section{Gestion des aires protégées pour la biodiversité}

Pour bien gérer les aires protégées, il faut les considérer comme un système intégré ayant un objectif à l'échelle du système, comme l'intégrité écologique. La gestion pratique de l'intégrité écologique passe souvent par la gestion de la diversité biologique (le plus souvent des espèces) et des fonctions écologiques. À l'échelle mondiale, les aires protégées constituent un outil essentiel pour maintenir la biodiversité. Cette section couvre les principes de gestion pour la conservation de la biodiversité dans les aires protégées aux trois niveaux : génétique, espèces et écosystèmes. Des exemple d'études de cas sont donnés pour chaque niveau de la biodiversité. De nombreuses autres études de cas sont disponibles en ligne ou en contactant des spécialistes des aires protégées de votre région.

\section{Gestion des aires protégées pour la diversité génétique}

Les aires protégées sont souvent établies pour conserver des caractéristiques uniques ou pour conserver des écosystèmes et des espèces représentatifs. Elles sont rarement établies ou conçues en ayant explicitement des aspects génétiques à l'esprit, même si la diversité génétique représente l'élément constitutif de l'évolution et de l'adaptation (Hughes et al., 2008). Une réduction de la diversité génétique limite le potentiel d'adaptation d'une population, et est souvent liée à une réduction de la condition physique (Frankham, 2005 ; Mattila et Seeley, 2007). Une réduction de la condition écologique au niveau individuel ajoute aux défis déjà rencontrés par les populations petites et isolées, contribuant à ce que l'on appelle le "vortex d'extinction " (Gilpin et Soulé, 1986 ; Caughley, 1994 ; Fagan et Holmes, 2006). Il a même été démontré que la diversité génétique a des effets importants sur les processus écologiques, tels que la productivité primaire (Hughes et al., 2008).

\section{Meilleures pratiques de gestion de la diversité génétique}

Maintenir la diversité génétique et éviter le vortex d'extinction sont des défis majeurs pour de nombreuses petites aires protégées isolées. Les solutions possibles incluent :

- Augmenter la taille effective de l'habitat de l'aire protégée afin qu'elle puisse contenir plus d'individus d'une espèce donnée. Cela peut se faire par l'acquisition de terres ou la restauration écologique (étude de cas 21.2).
- Les petites populations peuvent être augmentées par la translocation d'individus provenant de populations plus grandes et plus saines, afin d'augmenter la taille et la diversité génétique de la population locale (Bouzat et al., 2009). Il s'agit d'un principe bien établi, avec de nombreux exemples à travers le monde, comme la réintroduction de tigres (Panthera tigris tigris) dans le parc national de Sariska, en Inde, de tamarins-lions dorés (Leontopithecus rosalia) dans les forêts atlantiques du Brésil, ou de tétras des prairies (Tympanuchus cupido pinnatus) aux États-Unis (étude de cas 21.3).

- Travailler à l'échelle des paysages et à l'échelle régionale pour assurer la connectivité écologique entre des aires protégées distinctes, et assurer l'intégration des populations utilisant les paysages de travail autour des aires protégées. La connectivité augmente la taille effective de la population et permet le flux génétique entre les aires protégées et naturelles (di Minin et al., 2013 ; Sawaya et al., 2013 ; étude de cas 21.4).

\section{Gestion des aires protégées pour la diversité des espèces autochtones}

De nombreuses aires protégées sont actuellement gérées pour conserver des espèces ou des groupements d'espèces rares et endémiques. La gestion des aires protégées pour la diversité des espèces autochtones peut être très difficile, du fait que différentes espèces nécessitent souvent des mesures de gestion très différentes. Lorsque les ressources financières et humaines sont limitées, le défi consiste à décider des actions à cibler. Voici un bref aperçu de plusieurs options, telles qu'une gestion mettant l'accent sur des espèces clés, les niveaux trophiques, les pollinisateurs, et des espèces ou populations rares.

\section{Espèces clés}

L'influence des espèces sur les écosystèmes varie (Simberloff, 1998). Quelques espèces ont des effets disproportionnés par rapport à leur taille et à leur abondance sur la structure et les processus de l'écosystème et, par conséquent, sur la composition des espèces (Mills et al., 1993). Ces espèces sont décrites comme des "espèces clés ". Ce terme a été inventé en 1969 par Robert T. Paine, dont les recherches ont montré que l'élimination d'une seule espèce d'étoiles de mer avait un effet significatif sur un écosystème de bancs de sable dans l'État de Washington, aux États-Unis. Une fois l'étoile de mer disparue, la zone de marée a connu une domination par les moules, habituellement proies de l'étoile de mer, qui, à leur tour, ont déplacé d'autres espèces et réduit la diversité spécifique de l'écosystème (Paine, 1969). 


\section{Étude de cas 21.2 Augmenter la taille des aires protégées pour la diversité génétique}

En général, plus une aire protégée est petite, plus elle aura besoin d'une gestion active pour maintenir des composantes importantes de la biodiversité. Le parc Whiteman, dans la banlieue de Perth, en Australie, comprend la réserve Woodland, de 50 hectares, une installation électrifiée à l'épreuve des prédateurs, conçue pour la reproduction et pour fournir un habitat à des espèces rares et menacées, comme le bettongie à queue touffue (Bettongia pencillata) en danger critique d'extinction (Pacioni et al., 2011). Les gestionnaires s'inquiétaient de la viabilité à long terme des petites populations isolées d'espèces rares dans la réserve, et ont demandé à un généticien d'aider à élaborer un plan de gestion pour le bettongie à queue touffue. Le plan prévoyait une augmentation de la taille de la réserve de 50 à 200 hectares, ce qui, selon l'analyse, doublerait la durée pendant laquelle la colonie maintiendrait un niveau acceptable d'hétérozygotie (une mesure de la diversité génétique), une mesure clé de la santé génétique (Rafferty et Pacioni, 2012). Le plan recommandait également un programme régulier de supplémentation avec l'introduction de bettongie à queue touffue d'autres sites, afin de maintenir la diversité génétique au sein de la colonie.

\section{Étude de cas 21.3 Translocations pour le maintien de la diversité génétique}

Dans le Sud-Est de l'llinois, aux États-Unis, où presque toutes les prairies autochtones ont disparu, les gestionnaires ont essayé de maintenir le tétra des prairies (Tympanuchus cupido pinnatus), une espèce emblématique de ces régions, notamment dans deux petites aires protégées (Westemeier et al. 1998). Les efforts antérieurs comprenaient la restauration des prairies et l'augmentation de l'habitat de l'espèce, mais se sont révélés insuffisants pour rétablir la population (figure 21.4). Un suivi sur 35 ans a révélé que la taille de la population continuait de diminuer, de même que la fertilité, le succès de nidification et la diversité génétique (Bouzat et al., 1998). Préoccupés par le fait que la faible diversité génétique contribue à une réduction de l'aptitude évolutive de l'espèce, et exacerbe ainsi les effets négatifs de la petite taille de la population, les gestionnaires ont mis en place un programme de translocation, à partir d'oiseaux de populations plus grandes et plus saines, situées plus à l'Ouest (Westemeier et al. 1998). Les translocations se sont avérées fructueuses en ce sens qu'elles ont restauré la diversité génétique, contré les effets de la dépression de consanguinité, et conduit à une augmentation de l'adaptabilité évolutive (viabilité des œufs et succès de nidification) et, finalement, à une augmentation de la viabilité à long terme de la population (Bouzat et al., 2009). Cela illustre l'importance de gérer spécifiquement la diversité génétique afin de maintenir la biodiversité dans les aires protégées.

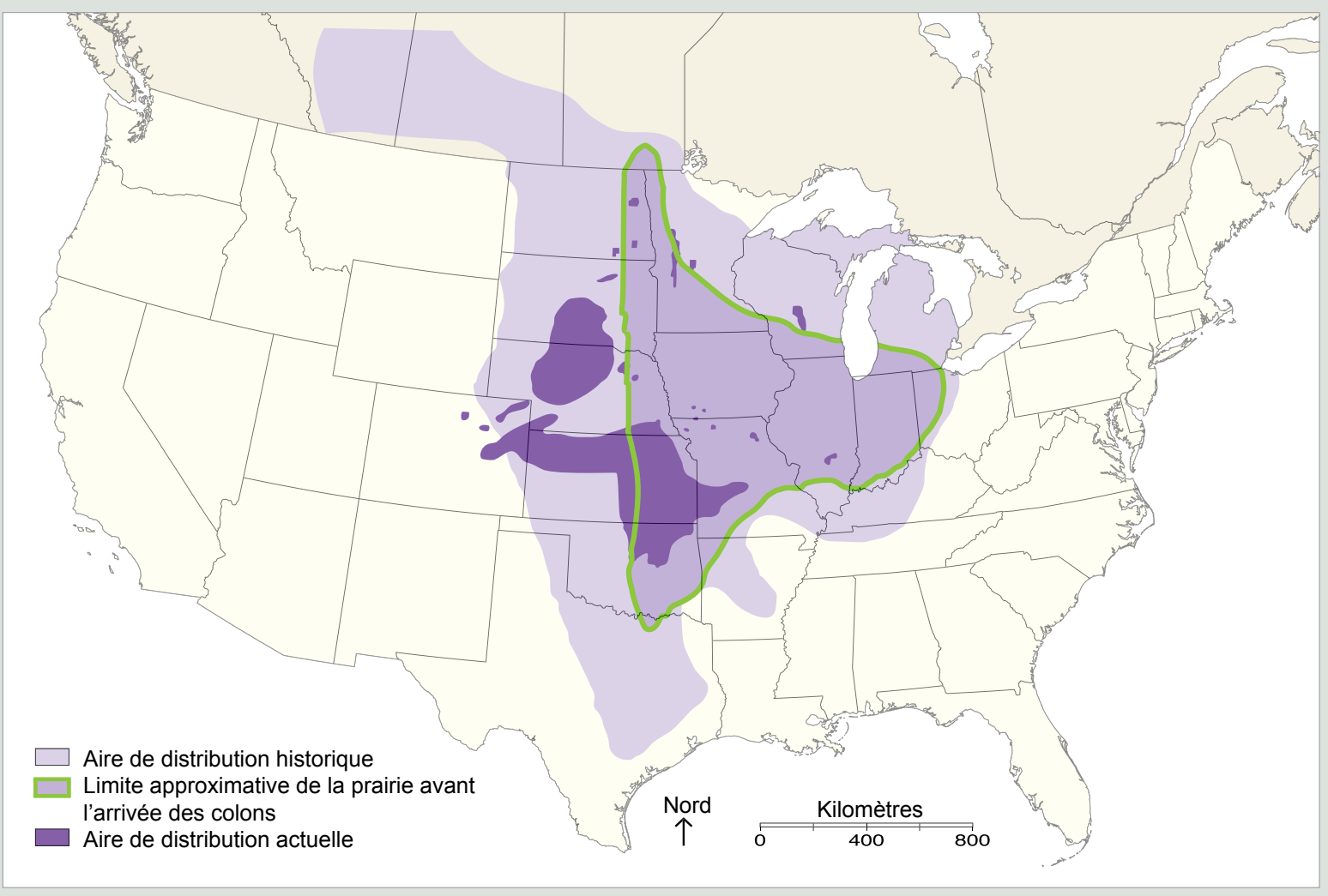

Figure 21.4 Répartition actuelle et historique du tétras des prairies (Tympanuchus cupido pinnatus) aux États-Unis

Source : Modifié de W. Daniel Svedarsky, Northern Prarie Wildlife Research Centre, Jamestown, ND, États-Unis 


\section{Étude de cas 21.4 Rétablir la connectivité dans le parc national de Banff pour maintenir la diversité génétique}

Le parc national de Banff abrite les paysages spectaculaires et la mégafaune des montagnes Rocheuses, attirant plus de visiteurs que tout autre parc national au Canada. II est coupé en deux par la route Transcanadienne et la ligne principale du chemin de fer Canadien Pacifique, deux voies de transport extrêmement fréquentées, qui fragmentent les habitats naturels du parc et entraînent une mortalité faunique importante (Clevenger et Sawaya, 2010). Afin de rétablir la connectivité et le flux génétique à travers les corridors de transport, Parcs Canada a construit 38 passages souterrains et six passages surélevés pour la faune, et installé des clôtures le long de la route (Clevenger et al., 2009). Environ 30 ans de suivi ont permis d'enregistrer plus de 120000 traversées, y compris de la plupart des grands mammifères : loups gris (Canis lupus), coyotes (Canis latrans), couguars (Puma concolor), cerfs (Odocoileus virginianus), wapitis (Cervus canadensis), orignaux (Alces alces), grizzlis (Ursus arctos horribilis) et ours noirs (Ursus americanus). Au cours des dernières années, des chercheurs ont testé des techniques non invasives de prélèvement d'ADN afin d'évaluer les effets génétiques et démographiques des passages fauniques dans le parc national de Banff (Sawaya et al., 2013). Bien qu'il n'existe actuellement aucune preuve empirique indiquant un flux génétique important à travers les passages surélevés, les passages souterrains ou le long des corridors (Corlatti et al., 2009), il semble probable que la lutte contre les effets de la fragmentation puisse contribuer à améliorer la diversité génétique, la viabilité des populations et, en fin de compte, la biodiversité dans les aires protégées (van der Ree et al., 2009).

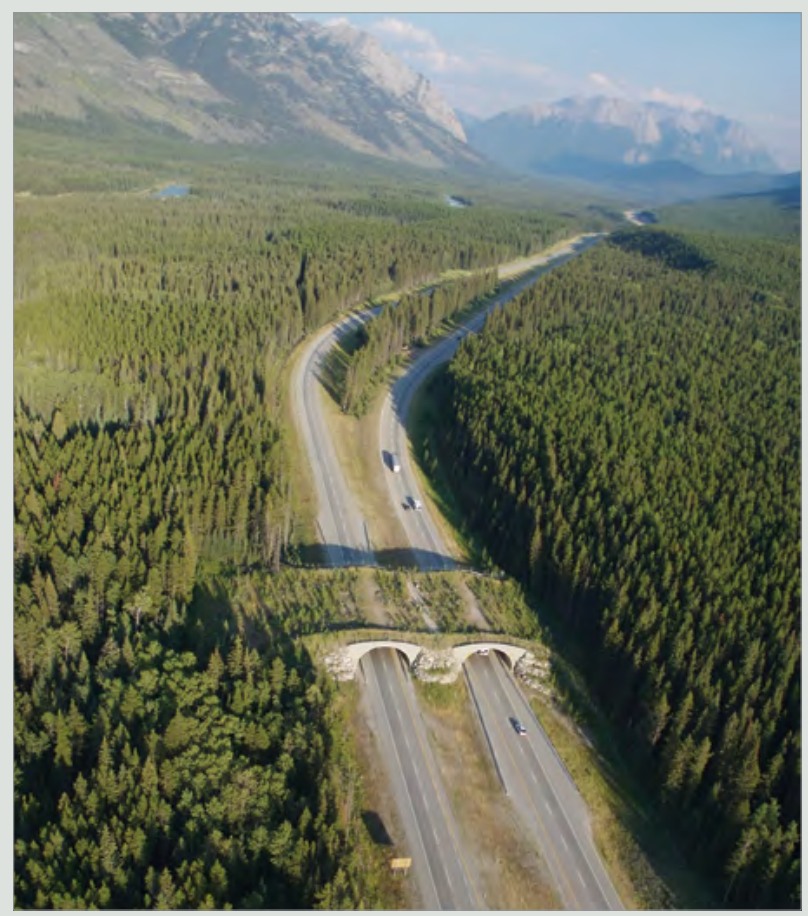

Passage faunique sur la route Transcanadienne dans le parc national de Banff, Canada

Source : Parcs Canada
De même, le pic de Magellan (Campephilus magellanicus) est considéré comme une espèce clé, parce qu'il aide à créer des structures d'habitat utilisées par huit autres espèces d'oiseaux et un mammifere, dans le parc national Nahuel Huapi, en Argentine (Ojeda, 2007). L'exploitation des forêts méridionales de hêtres, en Argentine et au Chili, a entrainé une diminution de l'abondance du pic, ainsi que des impacts négatifs pour les espèces associées. Une leçon clé de ces études est que les gestionnaires doivent comprendre les rôles des espèces individuelles afin de gérer, comprendre et restaurer les assemblages écologiques.

\section{Niveaux trophiques et cascades trophiques}

Un niveau trophique fait référence à la position qu’occupe une espèce dans la chaine alimentaire. Dans sa forme la plus simple, la chaîne alimentaire comprend les producteurs (par exemple, les plantes ou les algues), les consommateurs (par exemple, les herbivores et les carnivores) et les décomposeurs (par exemple, les bactéries et les champignons), l'énergie étant transférée le long de la chaîne (Pimm, 1982). Les écosystèmes réels, bien sûr, sont beaucoup plus complexes, présentant souvent plusieurs niveaux trophiques dans plusieurs réseaux trophiques (Estes et al., 2011). La gestion des aires protégées exige une compréhension des niveaux trophiques inhérents, les flux d'énergie se produisant principalement entre les niveaux, et non à l'intérieur de ceux-ci, et la perturbation de ces flux pouvant conduire à des changements majeurs dans les écosystèmes. En règle générale, ces changements résultent de l'élimination ou de la (ré)introduction de prédateurs, libérant ou contrôlant (respectivement) des herbivores, entraînant souvent des changements spectaculaires dans la structure de l'écosystème et le cycle des nutriments (études de cas 21.5 et 21.6).

\section{Pollinisateurs}

Les grands carnivores ne sont pas les seuls taxons affectant les écosystèmes. Les pollinisateurs jouent également un rôle clé. Il existe environ 350000 espèces de plantes à fleurs (WCSP, 2008), et bien que certaines dépendent du vent pour leur pollinisation, la grande majorité (plus de $85 \%$ ) dépend de la pollinisation par des animaux, ce qui souligne l'importance des pollinisateurs dans le maintien de la biodiversité (Ollerton et al., 2011). Les pollinisateurs comprennent environ 20000 espèces d'abeilles, ainsi que des mites, des papillons, des guêpes, des coléoptères, des mouches, des chauves-souris, des écureuils, des singes, des oiseaux, et bien d'autres. 


\section{Étude de cas 21.5 La loutre de mer et son impact sur les écosystèmes côtiers}

L'une des espèces clés les plus connues est sans doute la loutre de mer (Enhydra lutris), qui se nourrit d'une grande variété de proies. Plus important encore, elle consomme des oursins, qui se nourrissent de varech. Sans contrôle, les oursins peuvent décimer les paysages marins de varech, conduisant à des cascades trophiques. En aidant à contrôler l'abondance des oursins, les loutres de mer contribuent indirectement au maintien des écosystèmes de varech, qui fournissent nourriture et abri à de nombreuses autres espèces (Duggins, 1980). Malheureusement, les prélèvements de loutres de mer pour leur fourrure précieuse a entraîné la disparition de l'espèce de nombreuses parties de son aire de répartition, et son inscription comme espèce en voie de disparition aux États-Unis (Benz, 1996). Afin de restaurer cette importante espèce clé, de nombreuses tentatives de réintroduction ont été faites (Raesly, 2001). Plusieurs décennies après un effort de réintroduction dans la baie de Checleset, en ColombieBritannique, les loutres de mer sont de nouveau abondantes, et la baie abrite un écosystème de varech sain. Une réserve écologique a été créée spécifiquement pour protéger les loutres de mer et, par conséquent, l'écosystème de varech autochtone.

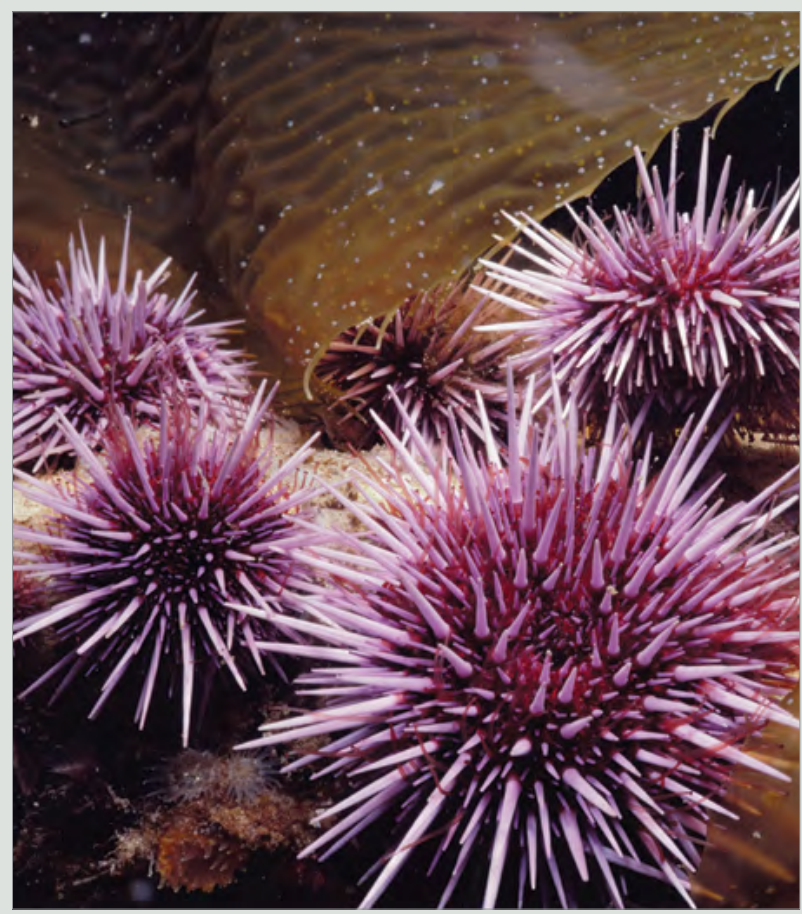

Oursin violet (Strongylocentrotus purpuratus)

Source : @ Jeff Rotman
Les aires protégées abritant des processus naturels limités et/ou isolées d'autres aires naturelles doivent souvent développer des techniques de gestion pratiques afin de maintenir la biodiversité autochtone. Par exemple, la réserve naturelle provinciale de Fish Point, située sur une île très développée du lac Ontario, est l'un des points les plus au sud du Canada et abrite plusieurs plantes rares.

Reconnaissant la réalité de la réduction de la diversité et de l'abondance des pollinisateurs régionaux, le plan de gestion du parc de, 2005 prévoyait la pollinisation manuelle, la collecte de graines et la multiplication assistée comme outils nécessaires pour aider à maintenir la diversité végétale du parc. Une étude menée en Afrique du Sud a révélé que, même dans des endroits riches en aires protégées et en biodiversité, les services de pollinisation diminuaient avec la distance par rapport aux aires naturelles. Ainsi, la biodiversité d'une aire protégée bénéficiera non seulement de la gestion des pollinisateurs, mais aussi des fermes voisines (Janzen, 1999 ; Carvalheiro et al., 2010 ; Chan et al., 2006).

Le maintien des pollinisateurs dans les aires protégées peut bénéficier à la biodiversité sur des distances considérables. Par exemple, le parc de conservation de l'île d'Indooroopilly, en Australie, offre une zone de repos importante pour trois espèces de roussettes. Les chauves-souris peuvent voler jusqu’à 100 kilomètres chaque nuit pour se nourrir, représentant des vecteurs importants pour le pollen, sur de longues distances. Par conséquent, les efforts de gestion du parc visant à conserver l'habitat des roussettes contribueront également au maintien de la diversité génétique et biologique dans l'ensemble de l'écosystème (Martin, 1990).

\section{Espèces rares}

La gestion des aires protégées donne souvent la priorité aux actions visant à maintenir l'intégrité écologique et profitant aux espèces communes, mais pas nécessairement aux espèces rares (Simberloff, 1998 ; Niemi et McDonald, 2004). Même si de nombreuses espèces sont rares, rares sont les espèces rares contribuant le plus à la biodiversité d'une région, comme l'avait déjà noté Charles Darwin. Par exemple, une analyse des espèces d'arbres dans les basses terres amazoniennes a révélé que la moitié des arbres appartenaient à 227 espèces " hyper-dominantes ", tandis que les autres étaient représentées par 11000 espèces (Steege et al., 2013). En d'autres termes, la grande majorité des espèces (plus de $98 \%$ ) étaient rares, du moins en termes d'abondance. Cependant, bon nombre des espèces représentées par proportionnellement peu d'arbres étaient répandues dans les basses terres et présentaient un nombre total élevé de troncs.

Cela soulève la question : que signifie rare et comment le mesurer ? Une espèce peut sembler rare dans un pays et être abondante dans un autre. Rabinowitz (1981) a suggéré que les espèces pourraient être considérées comme rares si elles 


\section{Étude de cas 21.6 Effet de cascade trophique à partir d'un prédateur supérieur}

Un exemple bien connu d'effet de cascade trophique a résulté de la réintroduction du loup gris dans le parc national du Yellowstone, aux États-Unis (Fortin et al., 2005). Le loup gris (46 individus) a été réintroduit dans le parc national du Yellowstone à partir de 1995, après avoir disparu en 1926. L'effet de cascade écologique résultant du rétablissement d'un prédateur supérieur est bien documentée, et illustre le rôle écologique critique joué par les prédateurs supérieurs (Ripple et al., 2001). En l'absence de loups et de chasse autochtone, la population de cerfs élaphe (Cervus elaphus) avait atteint des niveaux extrêmes, éliminant les populations de peupliers et réduisant la couverture latérale des saules. La réduction spectaculaire des peupliers et des saules avait entraîné l'élimination du castor (Castor canadensis), qui construisait des barrages dans une grande partie du parc, ce qui avait affecté le débit et provoqué l'érosion des cours d'eau. La réintroduction des loups a considérablement réduit le nombre de cerfs élaphe, les peupliers et les saules se sont rétablis, et le castor est réapparu dans certaines zones du parc. L'ensemble du processus de récupération prendra des décennies. Cet exemple illustre non seulement le rôle des grands prédateurs dans la régulation descendante des écosystèmes, mais aussi le rôle des aires protégées en tant que sites de recherche à long terme pour la compréhension écologique. Les stratégies d'aires protégées mettant l'accent sur le rôle clé que jouent les carnivores autochtones contribueront grandement au maintien de l'intégrité des écosystèmes et de la biodiversité.

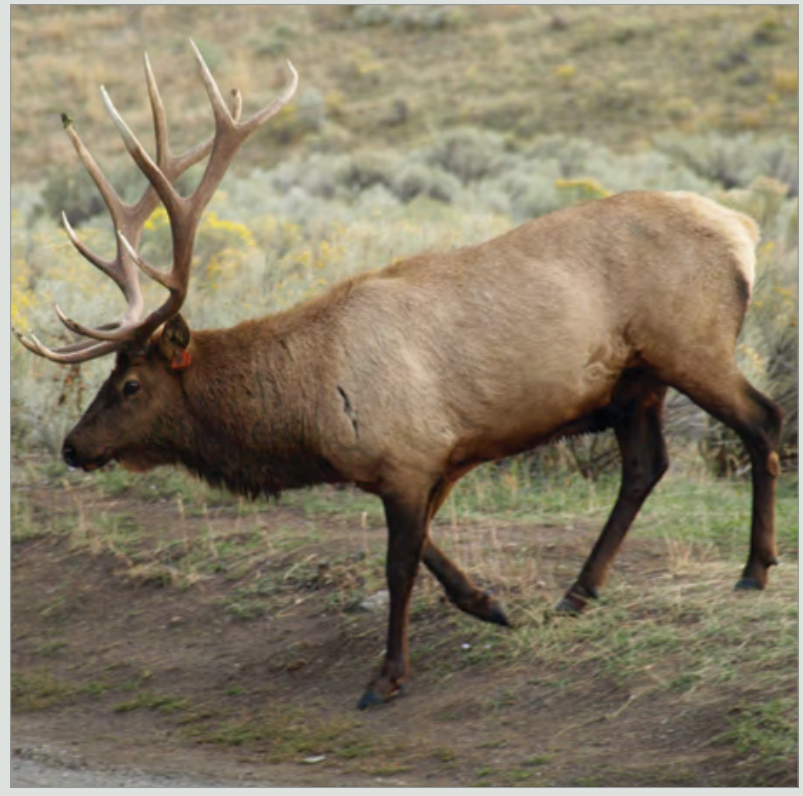

Cerf élaphe mâle (Cervus elaphus) à Mammoth, dans le parc national du Yellowstone, États-Unis d'Amérique

Source : Graeme L. Worboys présentaient une petite taille de population régionale, une distribution géographique restreinte ou une distribution d'habitat restreinte (spécificité élevée de l'habitat).

Les gestionnaires doivent également considérer à quel point une espèce est menacée, c'est à dire, son état de conservation. La Liste rouge de l'UICN des espèces menacées est basée sur une série de critères et de seuils précis, y compris la taille et la tendance de la population, l'aire de répartition géographique (mesurée par la zone d'occurrence et la zone d'occupation) ou la probabilité d'extinction, si suffisamment de données sont disponibles pour effectuer une analyse (IUCN, 2001). À partir d'une combinaison de critères, les espèces sont classées comme :

1. en danger critique d'extinction (CR) : risque extrêmement élevé d'extinction à l'état sauvage ;

2. en danger $(\mathrm{EN})$ : risque élevé d'extinction dans la nature ;

3. vulnérable (VU) : risque élevé de mise en danger dans la nature;

4. quasi menacée (NT) : susceptible de devenir en danger dans un proche avenir ;

5. préoccupation mineure (LC) : risque le plus faible, lorsque l'espèce ne correspond à aucune catégorie de risque plus élevée.
En 2012, la Liste rouge de l'UICN incluait près de 4000 espèces classées en danger critique d'extinction, 5766 en danger d'extinction, et plus de 10000 espèces vulnérables, bien que la plupart des groupes non vertébrés n'aient pas encore été évalués. Étant donné que de nombreuses espèces sont rares localement mais encore répandues, les gestionnaires doivent parfois utiliser des réseaux d'aires protégées pour maintenir les populations. Souvent, les aires protégées sont trop petites pour conserver un nombre viable d'individus de l'espèce, et un réseau d'aires protégées est nécessaire. Les gestionnaires d'aires protégées individuelles devront être conscients de ce que font leurs pairs dans d'autres aires protégées d'une même écorégion (Noss, 1983). Cela a été reconnu en Afrique du Sud, par exemple, où l'objectif d'une approche en réseau a été consacrée dans la législation, "dans le but d'assurer la création, le développement et la gestion efficace d'un réseau d'aires formellement protégées afin de conserver la biodiversité autochtone, des échantillons représentatifs d'écosystèmes naturels et des habitats d'espèces d'importance critique ou menacées " (North West Parks And Tourism Board Act 1997).

\section{Gestion des populations}

De nombreuses aires protégées ne sont pas assez grandes pour maintenir des populations viables de toutes les espèces. Dans ce cas, les gestionnaires doivent faire plus 
que protéger un site, et s'appuyer sur des processus naturels pour maintenir les espèces (Gurd et al., 2001 ; Landry et al., 2001 ; Deguise et Kerr, 2006). Par conséquent, une gestion active est souvent nécessaire. En règle générale, plus la réserve est petite, plus une gestion active sera nécessaire (MacKinnon et al., 1986). Cela inclut parfois la nécessité de prendre des mesures de gestion des populations, afin de faire face aux fluctuations de ces populations, des métapopulations et/ou d'assurer la viabilité des populations.

Dans une certaine mesure, l'abondance de toutes les populations sauvages fluctue en fonction des taux de natalité et de mortalité, des changements dans les ressources, de la température et des précipitations, de la prédation, des maladies et d'événements stochastiques (Boyce et Daley, 1980). En général, il existe quatre types de fluctuations de population :

1. Stable : lorsque les populations fluctuent légèrement au-dessus ou au-dessous de la capacité de charge.

2. Éruptive : lorsqu'une population normalement stable connaît une forte augmentation d'abondance en raison d'une augmentation temporaire de la capacité de charge.

3. Irrégulière : lorsqu'une population fluctue pour une raison non identifiée.

4. Cyclique : lorsqu'une population fluctue avec une fréquence régulière. Cela inclut les espèces suivant des cycles prédateur-proie.

Outre les fluctuations dans le temps, les populations peuvent varier d'un espace à l'autre, en particulier dans les paysages hétérogènes (Tilman et Kareiva, 1997). Il est donc difficile d'établir une population de référence pour une espèce spécifique, en l'absence de données à long terme et réparties dans l'espace. De plus, il est souvent difficile de distinguer les effets des activités humaines sur les populations d'espèces des fluctuations naturelles (Pechmann et al., 1991).

En l'absence de processus naturels et/ou de prédateurs, la taille de la population d'une espèce peut croître de façon incontrôlée, potentiellement jusqu'à ce qu'elle atteigne la capacité de charge du site, définie comme le nombre d'individus qu'une zone peut supporter, compte tenu des ressources disponibles (Stokes, 2012). Des populations anormalement abondantes peuvent finir par épuiser les ressources locales, entraînant un crash de population, comme dans le cas des éléphants dans certaines aires protégées africaines (Whyte, 2007), ou des conflits avec les voisins humains. Par exemple, un grand nombre de wapitis ont trouvé refuge contre les loups gris du parc national dans la ville de Banff, au Canada. Bien qu'il s'agisse d'animaux majestueux, ils peuvent également être très dangereux pour les humains, en particulier pendant la saison du rut. Suite à

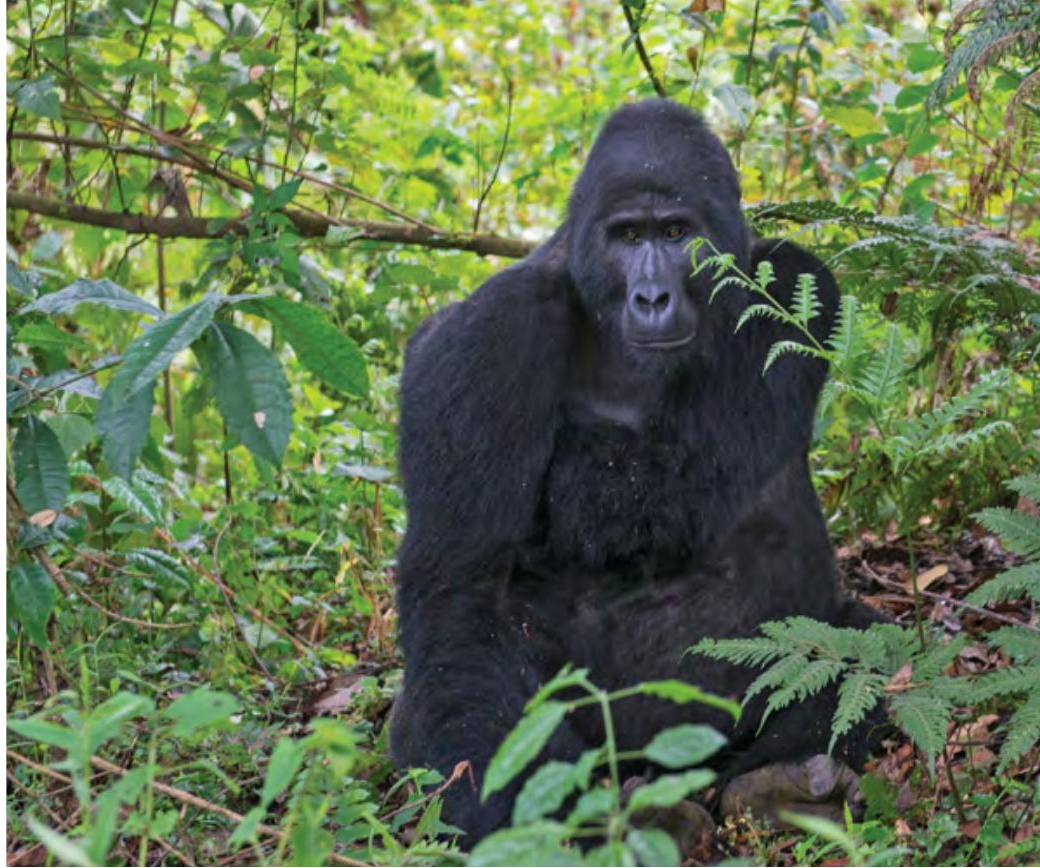

Gorille de montagne (Gorilla beringei beringei), une espèce en danger, dans l'impénétrable parc national de Bwindi, Ouganda

Source : Stuart Cohen

une série d'incidents de conflit homme-faune, Parcs Canada a mis sur pied un comité consultatif communautaire sur les wapitis, afin d'élaborer des propositions visant à régler le problème. Les mesures de gestion ont débuté en 1999 dans le but de restaurer les processus écologiques naturels sur les terres adjacentes à la ville, et de réduire les conflits entre les wapitis et les humains. Les mesures mise en place incluaient le piégeage de plus de 200 wapitis habitués et leur réinstallation dans une autre vallée de montagne, un programme de conditionnement aversif continu pour encourager les wapitis à éviter la ville, et la restauration de corridors fauniques pour augmenter la prédation des wapitis près de la ville. Les résultats ont été encourageants, avec moins de conflits entre les wapitis et les humains et une amélioration des conditions de l'écosystème adjacent à la ville, comme une réduction du broutage (White et al., 2007).

Le Service des parcs nationaux des États-Unis (NPS, 2006) a élaboré une politique pour gérer les fluctuations des populations, clarifiant le désir de s'appuyer sur les processus naturels, mais stipulant les conditions dans lesquelles une intervention est justifiée, y compris lorsque :

1. l'intervention n'aura pas d'effets inacceptables sur les populations de l'espèce ou sur d'autres composantes et processus des écosystèmes qui les soutiennent ; et

2. la gestion de la population est nécessaire :

- parce que la fluctuation est le résultat d'influences humaines ;

- pour protéger les espèces rares ;

- pour protéger les humains et les biens. 


\section{Métapopulations}

Lors de la gestion des aires protégées pour la biodiversité, il est important de reconnaître que certaines espèces peuvent exister en tant que métapopulations. Une métapopulation comprend généralement des sous-populations distinctes, chaque sous-population ayant sa propre dynamique (taux de natalité, de mortalité, d'immigration et d'émigration) (Hanski et Simberloff, 1997). Les métapopulations ne doivent pas être confondues avec une seule population dont la distribution est simplement inégale, mais présentant la même dynamique. Il est important que les gestionnaires comprennent s'ils gèrent une seule population ou une métapopulation d'une espèce donnée (Chapman et al., 2003). L'implication pour la gestion des aires protégées est qu'il est nécessaire de permettre la connectivité entre les sous-populations, ainsi que des mesures de gestion spécifiques pour une sous-population.

\section{Viabilité des populations}

Lobjectif le plus souvent cité lors de la gestion des populations dans les aires protégées est sans doute celui d'une population "viable ». La viabilité est mieux comprise comme la probabilité qu'une espèce donnée persiste sur une période définie. La première analyse de viabilité des populations (AVP) est attribuée à Mark Shaffer, dans son calcul de la probabilité d'extinction des grizzlis (Ursus arctos horribilis) dans le parc national du Yellowstone (Shaffer, 1978) en 1978. Depuis, les AVP sont devenues plus sophistiquées et complexes, incorporant de nombreuses variables susceptibles d'affecter la viabilité d'une espèce (Gilpin et Soulé, 1986 ; Traill et al., 2010), et culminant dans des logiciels qui ont installé l'AVP sur les ordinateurs de nombreux biologistes de parcs (par exemple, RAMAS, VORTEX). L'utilisation des AVP pour les décisions de gestion des aires protégées n'est toutefois pas sans controverses (Flather et al., 2011). De grandes quantités de données sont nécessaires pour effectuer des AVP robustes, et en particulier des données de terrain intensives et spécifiques aux espèces (Beissinger et McCullough, 2002). En outre, les résultats des AVP ont généralement de larges intervalles de confiance, et sont donc sujets à d'importantes erreurs (Flather et al., 2011). Néanmoins, les AVP peuvent fournir un aperçu des décisions de gestion, à condition qu'elles soient utilisées avec prudence, et sont utiles pour déterminer quelles variables ont la plus grande influence sur la viabilité des espèces (Akçakaya et Sjögren-Gulve, 2000). Par exemple, une étude sur une population de zèbre de montagne dans la réserve naturelle du mont Gamka, en Afrique du Sud, a permis de déterminer que le brûlage dirigé fréquent de l'habitat préféré de l'espèce était l'une des mesures de gestion les plus importantes, susceptible d'améliorer la viabilité de cette espèce en voie de disparition (Watson et al., 2005).

\section{Gestion des menaces pesant sur les aires protégées}

À l'échelle mondiale, les principales causes de perte de biodiversité et d'extinction des espèces sont la perte et la fragmentation des habitats, les autres menaces majeures incluant la dégradation et la pollution des habitats, la surexploitation, l'impact des espèces exotiques envahissantes et, de plus en plus, les changements climatiques. Bien qu'il soit prouvé que le statut d'aire protégée puisse conférer une protection supplémentaire aux habitats naturels (Geldmann et al., 2013), de nombreuses aires protégées sont encore menacées par la perte et la dégradation de leurs habitats (étude de cas 21.7).

Au cours des deux dernières décennies, le Partenariat pour les mesures de conservation a élaboré un cadre commun pour identifier les menaces à la diversité biologique et aux processus écosystémiques (Margoluis et Salafsky, 1998 ; TNC, 2000, 2007 ; Salafsky et al., 2003, 2008 ; CMP, 2013). Ce cadre peut être appliqué à la gestion des aires protégées (encadré 21.4 et tableau 21.1). Dans le cas des aires protégées, il est important de distinguer les contraintes (qui sont souvent internes à l'aire protégée et peuvent nécessiter des travaux de restauration), des menaces directes (qui proviennent généralement de l'extérieur de l'aire protégée). Souvent, à l'échelle d'un site individuel, ou même d'un système, les gestionnaires d'aires protégées ne peuvent aborder que les menaces directes, plutôt que les causes profondes sous-jacentes. Cela est particulièrement vrai lorsque les menaces pesant sur les aires protégées sont

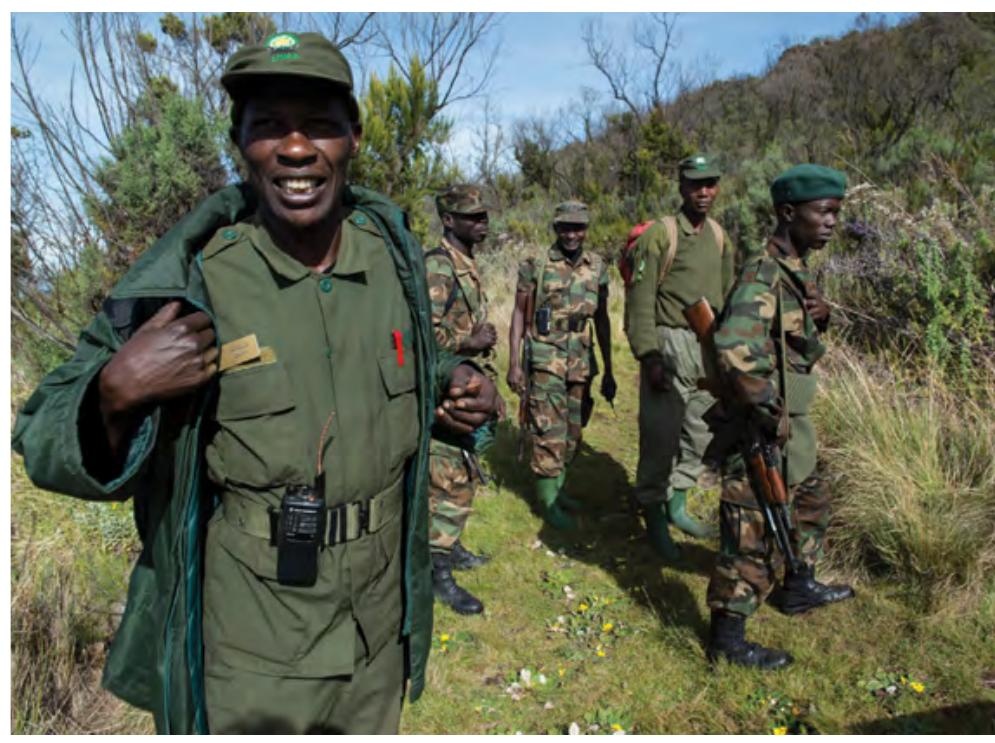

Rangers armés de l'Uganda Wildlife Authority, en patrouille dans le secteur du parc national du mont Elgon, pour protéger le parc contre le braconnage des espèces rares et menacées, et l'empiétement par les communautés voisines

Source : Stuart Cohen 


\section{Étude de cas 21.7 Aires protégées et pertes forestières}

Malgré le statut juridique des aires protégées, leur désignation ne garantit pas, en soi, la protection des écosystèmes. Bien que les aires protégées réduisent généralement la déforestation par rapport aux zones non protégées, des changements dans l'utilisation des terres peuvent encore y avoir lieu (Clark et al., 2008).

Une analyse de la déforestation dans les régions tropicales humides (Hansen et al., 2008) a montré qu'entre 2000 et 2005, environ 21 millions d'hectares de forêts tropicales humides ont été perdus dans le monde, soit une réduction de $2 \%$ du couvert forestier. Au cours de cette période, plus de 1,7 millions d'hectares ont été défrichés dans les aires protégées des régions tropicales humides (soit 0,81\% de la forêt qu'elles abritaient). À l'échelle mondiale, les aires plus strictement protégées (catégories | à || de I'UICN) présentaient des taux de perte de forêts tropicales humides plus faibles $(0,53 \%)$ que le réseau d'aires protégées dans son ensemble. Ceci a des implications pour la biodiversité et les changements climatiques. Sur la base des estimations de la déforestation, le Centre mondial de suivi de la conservation du Programme des Nations unies pour l'environnement (PNUE-WCMC) a calculé que la perte de forêts dans les aires protégées a contribué à hauteur de 990 mégatonnes d'équivalent carbone aux émissions mondiales de dioxyde de carbone entre 2000 et 2005, soit environ $3 \%$ des émissions totales de la déforestation tropicale (Campbell et al., 2008).

Dans une analyse systématique de l'efficacité des aires protégées pour réduire la perte de forêts et le déclin des populations d'espèces, Geldmann et al. (2013) ont conclu qu'il existe de bonnes preuves que les aires protégées ont conservé l'habitat forestier. Néanmoins, les données ne permettent pas de déterminer si les aires protégées ont été efficaces pour maintenir les populations d'espèces, bien que plus de résultats positifs que négatifs soient rapportés dans la littérature. Les liens de causalité entre apports de gestion et résultats de conservation dans les aires protégées sont rarement évalués dans la littérature. Dans l'ensemble, les données disponibles suggèrent que les aires protégées produisent des résultats positifs pour la biodiversité, mais peu de preuves empiriques sont disponibles concernant les conditions dans lesquelles les aires protégées réussissent ou ne parviennent pas à produire des résultats de conservation.

Les aires protégées visent, en fin de compte, à gérer des éléments clés (espèces, écosystèmes, possibilités récréatives et valeurs patrimoniales) et à les protéger des menaces. Dans un monde où les ressources humaines et financières sont limitées, les gestionnaires d'aires protégées ne peuvent pas nécessairement prendre en charge toutes les questions et tous les problèmes auxquels ces caractéristiques sont confrontées. Au lieu de cela, ils doivent être en mesure de concentrer leurs actions et leurs efforts de suivi sur les défis les plus importants. À cette fin, il est essentiel de pouvoir identifier et concevoir des stratégies réalistes pour contrer les menaces à des caractéristiques spécifiques dans les aires protégées individuelles. motivées par des politiques nationales ou locales et des facteurs sociaux et économiques sur lesquels un gestionnaire d'aires protégées peut avoir peu ou pas d'influence, par exemple, les politiques gouvernementales sur l'agriculture et les réseaux de transport (MacKinnon, 2005). D'autre part, les menaces directes à l'intérieur et à proximité immédiate des aires protégées peuvent être abordées par des mesures de gestion, par exemple, la lutte contre les espèces envahissantes, les conflits homme-faune et la gestion des visiteurs.

La conservation se fait finalement par le biais de " projets " de conservation (Salafsky et al., 2008), qui vont des efforts déployés par une petite communauté pour gérer ses zones de pêche traditionnelles à un programme de financement mondial pour protéger l'ensemble des océans de la planète. S'appuyant sur une analyse des termes utilisés par différents professionnels de la conservation, Salafsky et al. (2008) et le Partenariat pour les mesures de Conservation (CMP, 2013) ont proposé des définitions pour décrire les composantes générales d'un projet de conservation donné (encadré 21.4). Pour les gestionnaires d'aires protégées, la portée du projet est généralement définie par les limites de l'aire protégée et des zones tampons environnantes (voir le chapitre 13).

\section{Classification des menaces}

Le tableau 21.1 illustre la classification des menaces selon le Partenariat pour les mesures de conservation - UICN. La classification est construite de manière hiérarchique, en trois niveaux différents, analogues aux familles, genres et espèces du système linnéen. Le premier niveau est indiqué par des nombres entiers et un texte en gras, par exemple, «1. Développement résidentiel et commercial ". Le deuxième niveau est indiqué par des nombres décimaux et du texte normal, par exemple, «1.2. Zones commerciales et industrielles ». Le troisième niveau est indiqué en italique, par exemple, "Usines de fabrication ». La classification est conçue pour être complète, cohérente et exclusive pour les premier et deuxième niveaux, ce qui signifie que toutes les menaces possibles pour la biodiversité devraient pouvoir s'intégrer dans le système, chaque menace étant assignée à une seule catégorie. 


\section{Évaluation de l'ampleur des menaces pour la gestion des aires protégées}

La dernière étape de l'élaboration d'une approche systématique des menaces consiste à élaborer une méthode normalisée de mesure et de comparaison de l'ampleur des menaces. Si l'on souhaite simplement évaluer une menace spécifique pour une cible/caractéristique de conservation particulière ou une aire protégée au fil du temps, la mesure évidente consiste à évaluer directement et à réaliser un suivi de la magnitude de la menace en utilisant le meilleur indicateur quantitatif disponible, par exemple, le nombre d'incidents de braconnage d'éléphants, ou le pourcentage de forêt de la zone tampon perdu au profit du déplacement d'une frontière agricole. Toutefois, si l'on souhaite comparer les niveaux de menace combinés entre différentes cibles/ caractéristiques ou entre différentes aires protégées dans le temps et dans l'espace, une méthodologie plus complexe s'impose.

Les évaluations de l'ampleur des menaces sont importantes pour un certain nombre de tâches clés entreprises par les gestionnaires d'aires protégées. En particulier, sans mesures communes des menaces, il est difficile pour les gestionnaires d'aires protégées de :

- établir des priorités : comparer les aires protégées au sein d'un réseau global, établir des priorités pour l'investissement de ressources et planifier les sites prioritaires devant être gérés immédiatement et ceux pouvant être reportés à plus tard ;

- élaborer des stratégies efficaces : choisir les menaces à aborder dans une aire protégée donnée, comparer l'effet de levier potentiel obtenu en utilisant différentes stratégies et décider desquelles utiliser ;

- mesurer les conditions et l'efficacitéde la conservation: déterminer et comparer les changements dans les conditions des menaces à un endroit, au fil du temps, et déterminer l'efficacité relative des différentes mesures de conservation par rapport aux objectifs relatifs aux menaces ;

- apprendre de l'expérience : comparer les expériences d'un gestionnaire avec celles des autres, ce qui constitue le fondement de tout type d'apprentissage systématique sur la façon de contrer efficacement, et à moindre coût, chaque type de menace.

À la fin des années 1990 et au début des années 2000, un certain nombre d'organisations de conservation ont commencé à mettre au point des méthodes systématiques pour évaluer l'ampleur des menaces de manière plus

\section{Encadré 21.4 Définitions clés pour comprendre les menaces aux écosystèmes}

- Cibles de biodiversité : entités biologiques (espèces, communautés ou écosystèmes) qu'un projet tente de conserver (par exemple, une population d'une espèce spécifique de poisson ou un écosystème forestier). Certains professionnels incluent également des phénomènes et des processus écologiques et évolutifs en tant que cibles (par exemple, régime de feu, migration saisonnière, flux génétique).

- Objectifs de bien-être humain : composantes du bien-être humain affectées par les conditions des cibles de conservation de la biodiversité. II peut s'agir, par exemple, de moyens de subsistance humains issus de l'utilisation de ressources biologiques ou de valeurs spirituelles issues de systèmes naturels.

- Stress : attributs de l'écologie d'une cible de conservation altérés directement ou indirectement par les activités humaines (par exemple, réduction de la taille d'une population ou fragmentation des habitats forestiers). Le stress n'est pas une menace en soi, mais plutôt un état dégradé ou un « symptôme " de la cible résultant d'une menace directe.

- Menaces directes : activités ou processus humains immédiats ayant causé, causant ou susceptibles de causer la destruction, la dégradation et/ou la détérioration des cibles de biodiversité (par exemple, la pêche ou l'exploitation forestière non durable). Les menaces peuvent être passées (historiques), en cours et/ou susceptibles de se produire à l'avenir. Les phénomènes naturels peuvent également être considérées comme des menaces directes dans certaines situations.

- Facteurs sous-jacents : facteurs ultimes, généralement socioéconomiques, politiques, institutionnels ou culturels, qui permettent ou renforcent l'apparition ou la persistance de menaces directes immédiates (par exemple, les politiques agricoles gouvernementales ou les forces du marché qui augmentent l'expansion des terres agricoles ou la surexploitation des ressources telles que la pêche).

- Actions de conservation : interventions entreprises par le personnel du projet ou les partenaires, visant à atteindre les objectifs du projet et les buts ultimes de conservation (par exemple, réintroduction d'une espèce en voie de disparition ou création d'une aire protégée). Les actions peuvent être appliquées aux facteurs sous-jacents, aux menaces directes, ou directement aux cibles elles-mêmes.

- Équipes de projet : groupes de personnes impliquées dans la conception, la mise en œuvre, la gestion et le suivi des projets (par exemple, un partenariat entre une organisation non gouvernementale locale ou une communauté et le personnel d'un parc national). 
normalisée (par exemple, Salafsky et Margoluis, 1999 ; TNC, 2000 ; Ervin, 2002 ; WCS, 2002). Au milieu des années 2000, un groupe de travail du CMP a analysé ces différents systèmes, et les a utilisés pour créer une méthodologie standard d'évaluation des menaces, devenue depuis la base de la méthodologie « Évaluation simple des menaces » du logiciel Miradi (Miradi, 2007).

Tableau 21.1 Catégories de menaces, et quelques exemples de la classification actuelle des menaces du Partenariat pour les mesures de conservation-UICN

Menaces par niveau de classification

1. Développement résidentiel et commercial

\subsection{Logements et zones urbaines}

Zones urbaines, banlieues, villages, maisons de vacances, zones commerçantes, bureaux, écoles, etc.

\section{Agriculture et aquaculture}

\subsection{Aquaculture marine et d'eau douce}

Aquaculture de crevettes ou de poissons à nageoires, étangs piscicoles dans les fermes, élevages de saumons, bancs de mollusques ensemencés, herbiers marins artificiels.

\section{Production d'énergie et exploitation minière}

3.1. Forage pétrolier et gazier

Puits de pétrole, forage de gaz naturel en haute mer.

\section{Corridors de transport et de service}

\subsection{Routes et voies ferrées}

Routes, routes secondaires, routes forestières, ponts et chaussées, mortalité animale due aux véhicules, clôtures associées aux routes, voies ferrées.

\section{Utilisation des ressources biologiques}

\subsection{Chasse et collecte d'animaux terrestres} Chasse à la viande de brousse, chasse aux trophées, piégeage de fourrures, collecte d'insectes, récolte de miel ou de nids d'oiseaux, lutte contre les prédateurs, lutte antiparasitaire, persécution.

\section{Intrusions et perturbations humaines}

\subsection{Activités de loisirs}

Véhicules tout-terrains, bateaux à moteur, jet skis, motoneiges, avions ultralégers, bateaux de plongée, observation des baleines, VTT, randonneurs, ornithologues, skieurs, etc.

\section{Modifications du système naturel}

\section{Définition}

Établissements humains ou autres utilisations des terres non agricoles ayant une empreinte importante.

Villes, villages et établissements humains, y compris les infrastructures non destinées au logement mais généralement intégrées à un logement.

Menaces provenant de l'agriculture et de l'élevage, en raison de l'expansion et de l'intensification de l'agriculture, y compris la sylviculture, la mariculture et l'aquaculture.

Animaux aquatiques élevés en un seul endroit sur des ressources d'élevage ou non locales, et élevage piscicoles ouverts.

Menaces liées à la production de ressources non biologiques.

Exploration, développement et production de pétrole et autres hydrocarbures liquides.

Menaces provenant de longs corridors de transport étroits et des véhicules qui les utilisent, y compris la mortalité faunique associée.

Transport de surface sur routes et voies réservées.

Menaces provenant de l'utilisation de ressources biologiques "sauvages ", y compris les effets délibérés et non intentionnels de la récolte, ainsi que la persécution ou le contrôle d'espèces spécifiques.

Tuer ou piéger des animaux sauvages terrestres ou récolter des produits animaux à des fins commerciales, récréatives, de subsistance, de recherche ou culturelles, ou pour des raisons de contrôle ou de persécution, y compris la mortalité accidentelle et les prises accessoires.

Menaces découlant d'activités humaines modifiant, détruisant ou perturbant les habitats et les espèces associés aux utilisations non consommatrices des ressources biologiques.

Personnes passant du temps dans la nature ou voyageant dans des véhicules en dehors des corridors de transport établis, généralement pour des raisons récréatives.

Menaces provenant d'actions transformant ou dégradant les habitats au service de la " gestion » des systèmes naturels ou semi-naturels, souvent pour améliorer le bienêtre humain. 
7.1. Feu et lutte contre le feu

Suppression des feux de forêt pour protéger les habitations, gestion inappropriée des feux de forêt, incendies agricoles non contrôlés, incendies criminels, feux de camp, feux de chasse.

\section{Espèces envahissantes et autres espèces et gènes problématiques}

8.1. Espèces exotiques/non autochtones envahissantes Bétail sauvage, animaux domestiques, moules zébrées, maladie hollandaise de l'orme ou mildiou du châtaignier, Miconia, introduction d'espèces pour la lutte biologique, champignon Chytride affectant les amphibiens en dehors de l'Afrique.

\subsection{Espèces autochtones problématiques}

Cerfs autochtones surabondants, algues surabondantes dues à la perte de poissons brouteurs autochtones, plantes autochtones s'hybridant avec d'autres plantes, peste affectant les rongeurs.

\section{Pollution}

\subsection{Effluents industriels et militaires}

Produits chimiques toxiques provenant d'usines, déversement illégal de produits chimiques, résidus miniers, arsenic provenant de mines d'or, fuites dans des réservoirs de carburant, PCB dans les sédiments fluviaux.

\section{Phénomènes géologiques}

10.2. Tremblements de terre/tsunamis

\section{Changements climatiques et phénomènes} météorologiques extrêmes

\subsection{Déplacement et altération des habitats}

Élévation du niveau de la mer, désertification, dégel de la toundra, blanchiment des coraux.

\subsection{Sécheresse}

Déficit grave de pluie, perte de sources d'eau de surface.

Source : Classification des menaces directes pour la biodiversté de l'UlC
Suppression ou augmentation de la fréquence et/ou de l'intensité du feu au-delà de sa gamme naturelle de variation.

Menaces provenant de plantes, d'animaux, d'agents pathogènes/microbes ou de matériel génétique non autochtone et autochtone ayant ou susceptibles d'avoir des effets nocifs sur la biodiversité, suite à leur introduction, propagation et/ou augmentation d'abondance.

Plantes, animaux, agents pathogènes et autres microbes nuisibles ne se trouvant pas, à l'origine, dans le ou les écosystèmes en question, et directement ou indirectement introduits et propagés par les activités humaines.

Plantes, animaux ou agents pathogènes nuisibles et autres microbes se trouvant, à l'origine, dans le ou les écosystèmes en question, mais qui ont connu un " déséquilibre » ou ont été « libérés » directement ou indirectement en raison d'activités humaines.

Menaces liées à l'introduction de matières ou d'énergie exotiques et/ou excédentaires provenant de sources ponctuelles et non ponctuelles.

Polluants d'origine hydrique provenant de sources industrielles et militaires, y compris l'exploitation minière, la production d'énergie et autres industries d'extraction de ressources, y compris les nutriments, les produits chimiques toxiques et/ou les sédiments.

Menaces découlant de phénomènes géologiques catastrophiques.

Tremblements de terre et événements associés.

Changements climatiques à long terme pouvant être liés au réchauffement de la planète et autres phénomènes climatiques ou météorologiques extrêmes, au-delà de la plage de variation naturelle, susceptibles d'anéantir une espèce ou un habitat vulnérable.

Changements majeurs dans la composition et l'emplacement des habitats.

Périodes où les précipitations tombent en dessous de la plage normale de variation.

\section{Méthode d'évaluation simple des menace de Miradi}

Le logiciel de gestion adaptative Miradi (Miradi, 2007) est conçu pour être utilisé pour évaluer l'impact d'une menace spécifique sur une cible de conservation donnée, à partir d'une combinaison de portée (étendue) et de gravité (intensité) qui, lorsque combinées, fournissent une indication de l'ampleur de la menace. Miradi utilise des échelles d'évaluation spécifiques à quatre niveaux pour chaque critère (très élevé, élevé, moyen et faible) liées, dans la mesure du possible, à des pourcentages spécifiques. Les seuils entre les critères sont conçus pour représenter à la fois des points de rupture significatifs sur le plan écologique et pratique entre les catégories. Le système d'évaluation des menaces de Miradi devrait généralement s'appliquer aux aires protégées de tous types et de toutes tailles. Cependant, il pourrait être nécessaire d'adapter le système pour gérer les évaluations de caractéristiques non liées à la conservation, 


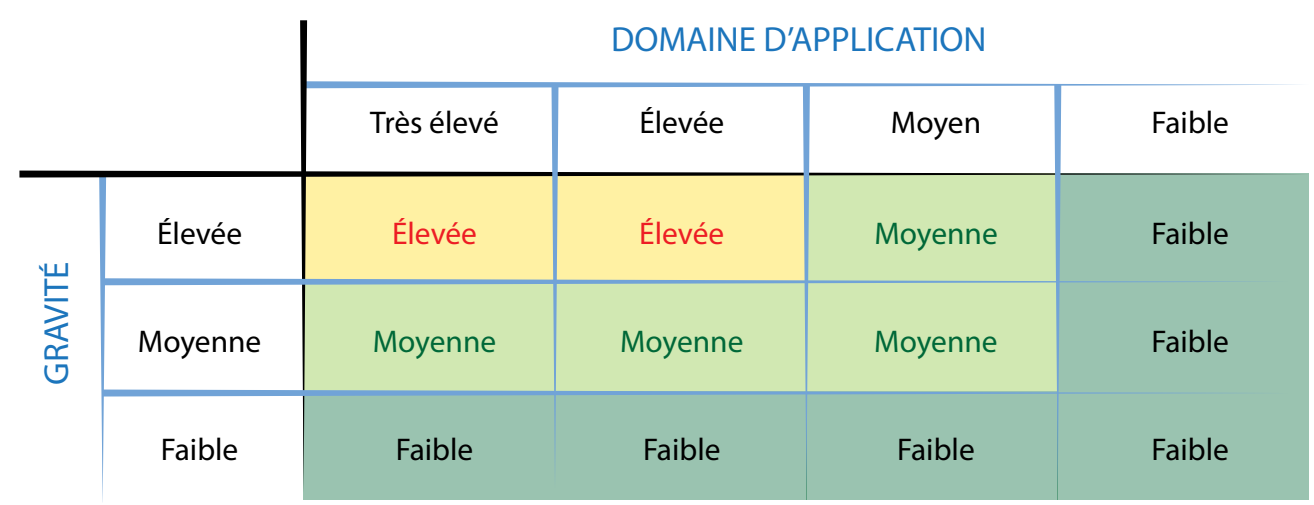

Figure 21.5 Évaluations combinées de la menace et de la gravité pour déterminer l'ampleur d'une menace Source : Miradi (2007)

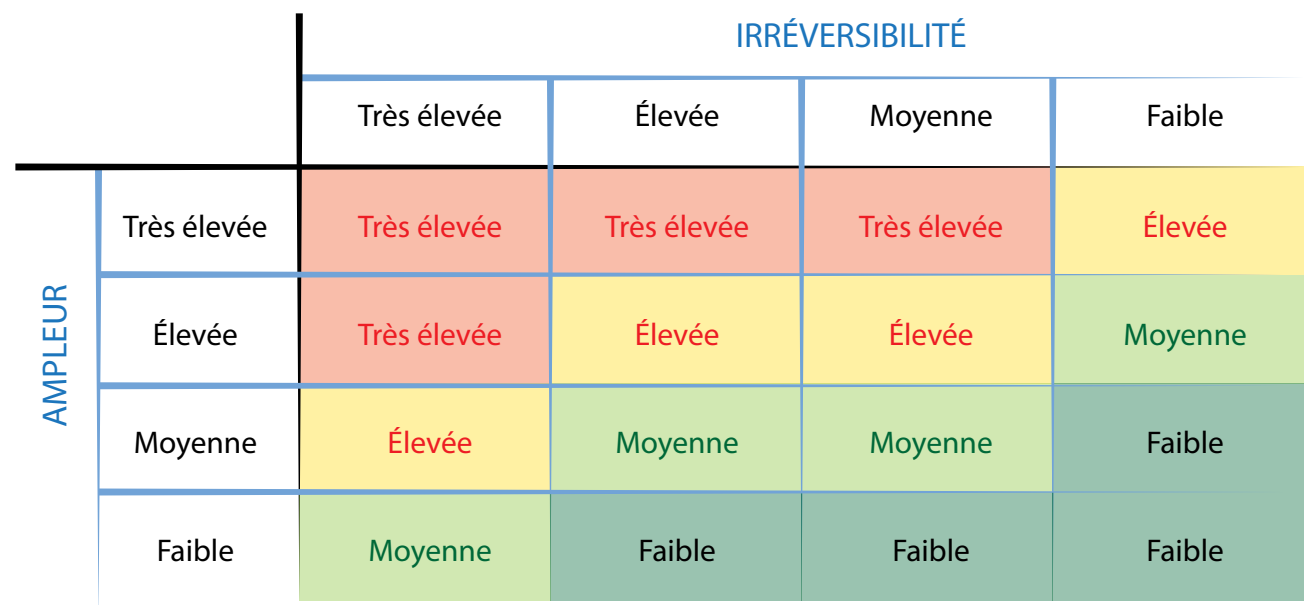

Figure 21.6 Évaluations combinées de l'irréversibilité et de l'ampleur de la menace visant à hiérarchiser les actions de gestion

Source : Miradi (2007)

\begin{tabular}{|c|c|c|c|c|c|c|c|}
\hline $\begin{array}{l}\text { Menaces / } \\
\text { valeurs }\end{array}$ & $\begin{array}{c}\text { Éléphant } \\
\text { d'Asie }\end{array}$ & $\begin{array}{l}\text { Écosystème } \\
\text { forestier }\end{array}$ & $\begin{array}{c}\text { Corridor } \\
\text { fonctionnel }\end{array}$ & $\begin{array}{c}\text { Prairies du } \\
\text { Teraï }\end{array}$ & $\begin{array}{l}\text { Rhinocéros } \\
\text { indien }\end{array}$ & Tigre & $\begin{array}{c}\text { Évaluation } \\
\text { résumée de la } \\
\text { menace }\end{array}$ \\
\hline $\begin{array}{l}\text { Pâturage du } \\
\text { bétail }\end{array}$ & & Élevée & Faible & Élevée & & & Élevée \\
\hline Empiètement & & $\begin{array}{l}\text { Très } \\
\text { élevée }\end{array}$ & $\begin{array}{l}\text { Très } \\
\text { élevée }\end{array}$ & Élevée & & & $\begin{array}{l}\text { Très } \\
\text { élevée }\end{array}$ \\
\hline $\begin{array}{l}\text { Collecte de bois } \\
\text { de chauffage }\end{array}$ & & Élevée & Moyenne & & & & Moyenne \\
\hline Braconnage & & & & & $\begin{array}{l}\text { Très } \\
\text { élevée }\end{array}$ & $\begin{array}{l}\text { Très } \\
\text { élevée }\end{array}$ & $\begin{array}{l}\text { Très } \\
\text { élevée }\end{array}$ \\
\hline $\begin{array}{l}\text { Exploitation } \\
\text { forestière }\end{array}$ & & Faible & Faible & & & & Faible \\
\hline $\begin{array}{l}\text { Surexploitation } \\
\text { de produits non }\end{array}$ & & Faible & Faible & Faible & & & Faible \\
\hline $\begin{array}{l}\text { Résumé de } \\
\text { l'évaluation de la } \\
\text { cible }\end{array}$ & Aucune & Élevée & Élevée & Élevée & Élevée & Élevée & $\begin{array}{l}\text { Très } \\
\text { élevée }\end{array}$ \\
\hline
\end{tabular}

Figure 21.7 Évaluation résumée de la menace pour un exemple d'écosystème

Source : Miradi (2007) 
ainsi que les contraintes posées par des menaces telles que les changements climatiques. La combinaison des évaluations de portée et de gravité produit une évaluation globale de l'ampleur de la menace (figure 21.5). Il est également utile de considérer l'irréversibilité (la mesure dans laquelle les effets d'une menace peuvent être inversés) en combinaison avec l'ampleur, afin de comparer ou de hiérarchiser les menaces dans le cadre des mesures de gestion (figure 21.6). Les effets d'une menace sur plusieurs cibles, ou de menaces multiples sur une seule cible, peuvent être combinés ou regroupés à l'aide de divers systèmes basés sur des règles (pour plus de détails, voir Miradi, 2007). On obtient ainsi un tableau récapitulatif final des menaces (figure 21.7).

La communauté de conservation de la biodiversité a fait de grands progrès, au cours des dernières années, dans le développement de méthodes standardisées permettant de définir et de mesurer les menaces pour les espèces et les écosystèmes. Le potentiel d'utilisation de ces outils dans la gestion des aires protégées est important, mais des modifications seront nécessaires pour optimiser ces méthodes en fonction des besoins spécifiques des gestionnaires d'aires protégées.

\section{Suivi et évaluation des conditions écologiques des aires protégées}

Dans la présente section, nous considérons le suivi comme englobant à la fois l'inventaire et le suivi. L'inventaire est la première étape essentielle, et le suivi consiste généralement en une série de mesures répétées du premier inventaire ou de certaines parties de celui-ci. Très peu d'aires protégées font un bon travail de suivi écologique, même si celui-ci est essentiel pour comprendre si l'aire protégée réussit à conserver la nature et à atteindre ses objectifs de conservation déclarés. De plus, un investissement dans le suivi évite les surprises et problèmes futurs insolubles. L'intégration du suivi à la gestion du parc devrait être considérée comme un élément fondamental de la gestion de celui-ci. À long terme, il peut permettre de faire des économies en évitant des projets de restauration coûteux (voir également le chapitre 28).

Même si aucun système de suivi n'y est actuellement en place, la plupart des aires protégées peuvent trouver des données utiles pour évaluer leurs conditions écologiques. Les visiteurs, le personnel, les scientifiques et les peuples autochtones et locaux font régulièrement des observations sur les terres et les eaux des aires protégées. Les systèmes de capteurs mondiaux, y compris les satellites et les stations météorologiques, effectuent continuellement des observations et, de plus en plus, des informations satellitaires gratuites sont disponibles (étude de cas 21.8). La première étape du développement d'un système de suivi de la diversité biologique et des processus écosystémiques consiste à cataloguer et à organiser ces données existantes, afin de fournir les meilleures preuves disponibles pour prendre des décisions de gestion.

Deux questions de suivi sont clés pour les aires protégées. Cette section vous aidera à comprendre et à élaborer des réponses à ces questions.

1. Quel sont les conditions écologiques : devons-nous prendre des mesures de gestion?

2. Les mesures de gestion ont-elles été efficaces?

Les gestionnaires veulent disposer de la meilleure information possible pour répondre à ces questions. Dans la pratique, cela constitue un défi, car ces deux questions sont complexes. Au départ, ne vous préoccupez pas trop du montant d'argent et de l'expertise disponibles pour l'observation de l'aire protégée. Quelques informations valent mieux qu'aucune, bien que les décisions de gestion doivent se fonder sur ce qui est connu et inconnu. Même des informations simples et bien organisées sont plus convaincantes que des informations déconnectées et mal documentées. La meilleure pratique consiste à travailler avec ce qui est disponible, et à établir des partenariats pour le suivi à long terme afin de compléter le suivi effectué par le personnel des aires protégées.

Le présent chapitre ne fournit pas de guide complet pour tous les éléments de conception d'un programme de suivi, ni pour la collecte et l'analyse des données. Les lecteurs sont invités à consulter des guides sur le sujet, tels que Lindenmeyer et Likens (2010) et Gitzen (2013). En outre, de nombreux sites web d'organismes chargés des aires protégées contiennent des détails sur leur suivi et protocoles de suivi, tels que le Programme d'inventaire et de suivi du Service des parcs nationaux des États-Unis (NPS, 2014). Enfin, un grand nombre de guides spécifiques aux taxons et aux écosystèmes sont disponibles en ligne. Cette section traite des considérations fondamentales pour une réflexion sur la conception et la mise en œuvre d'un programme de suivi de la biodiversité et des fonctions écosystémiques pour les aires protégées.

\section{Quoi observer?}

\section{Suivi des conditions}

Pour répondre à la question "Quelles sont les conditions écologiques : devons-nous prendre des mesures de gestion ? " la première exigence est de savoir ce qui devrait 


\section{Étude de cas 21.8 Suivi mondial des feux de forêt}

Les feux de forêt et les incendies dû à l'agriculture sur brûlis sont deux des causes les plus importantes de déforestation à Madagascar. L'utilisation de la télédétection par satellite pour détecter les incendies peut permettre aux gestionnaires d'aires protégées et autres forêts de réagir plus rapidement aux incendies illégaux. L'instrument MODIS (ModerateResolution Imaging Spectroradiometer / Spectroradiomètre à résolution moyenne) de la NASA, embarqué sur les satellites Aqua et Terra, fournit des données thermiques et infrarouges moyens quatre fois par jour, permettant la détection des incendies. Les données, cependant, doivent être interprétées et analysées, ce qui rend leur utilisation par les gestionnaires de terrain difficile. Afin de développer un produit plus convivial, Conservation International, l'Université du Maryland et le Département des forêts de Madagascar ont mis au point un système d'alerte incendie fournissant quotidiennement des alertes aux utilisateurs, par e-mail, en fonction de leur zone géographique d'intérêt.

À Madagascar, les alertes incendie fournissent au personnel des aires protégées et des forêts des informations précises et actualisées sur les incendies illégaux et les activités d'empiétement. Cela permet aux gestionnaires de terrain de réagir rapidement à l'empiètement, mais le système fournit également des statistiques de suivi précieuses pour surveiller la menace d'incendie sur différents sites. Les données d'incendies peuvent également être utilisées pour améliorer la compréhension des modèles de menaces pour les forêts à l'échelle nationale, et ont été utilisées pour guider l'élaboration de la stratégie nationale pour l'atténuation des changements climatiques par la Réduction des émissions issues de la déforestation et de la dégradation des Forêts (REDD).

Initialement, en 2002, le système d'alerte incendie envoyait aux utilisateurs enregistrés une simple liste des incendies détectés avec leur position exacte. Un système plus avancé, en 2007, permet maintenant aux utilisateurs de définir la fréquence des alertes et de recevoir des cartes adaptées à leurs domaines d'intérêt spécifiques, par exemple, des régions administratives, des parcs nationaux individuels ou les incendies affectant les forêts naturelles. Le système, connu sous le nom de Firecast, a depuis été étendu à la Bolivie, au Pérou et à l'Indonésie, et inclut désormais des alertes de prévision des risques d'incendie. Firecast est gratuit et accessible (Firecast, 2014).

- James Mackinnon

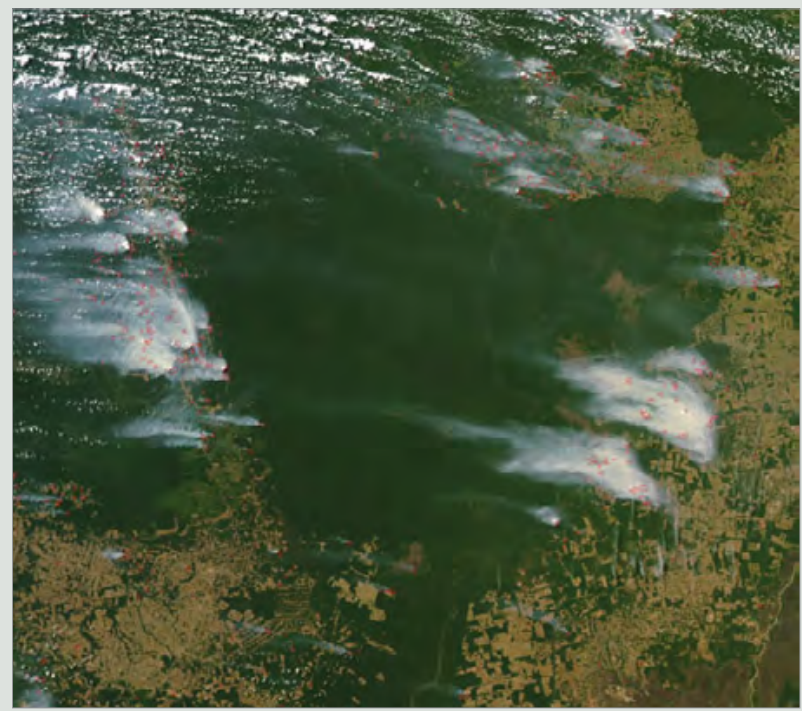

Incendies près du parc autochtone de Xingu, Brésil Source : Jacques Descloitres, équipe d'intervention rapide MODIS, NASA-Goddard Space Flight Center, <rapidfire.sci.gsfc.nasa.gov> être mesuré pour évaluer les conditions écologiques. Il est logique de commencer par évaluer les espèces et les processus mentionnés dans le document d'établissement ou les plans de gestion de l'aire protégée. Les espèces immédiatement identifiées avec le site et les processus écologiques, et maintenant son aspect caractéristique (par exemple, le feu sur la savane), sont susceptibles d'être le point de départ le plus important (voir la section «Évaluation des conditions de l'aire protégée : intégrité écologique " (ci-dessus). Les politiques et la législation de l'organisme chargé des aires protégées peuvent également fournir des conseils sur la sélection d'espèces et de processus spécifiques à surveiller, par exemple, les espèces rares et les principaux objectifs de conservation. Il est important de maintenir une approche systématique et impartiale du suivi afin d'éviter les préjugés des chercheurs. Les gestionnaires des aires protégées devront utiliser un cadre structuré pour sélectionner les indicateurs. Dans la plupart des cas, un ensemble d'indicateurs devra être choisi pour chacun des principaux écosystèmes d'une aire protégée, c'est-à-dire les forêts, les zones humides, les prairies, etc. Un exemple de modèle de suivi écologique utilisé par Parcs Canada, et incluant la biodiversité, les fonctions écosystémiques et les facteurs de stress connus comme éléments du cadre de suivi, est illustré dans le tableau 21.2.

Après avoir sélectionné une liste d'espèces, de fonctions et de menaces, les gestionnaires devront tenir compte du coût, en temps et en argent, de la mesure de ces différents aspects. Il existe souvent des façons de faire les choses à moindre coût pour obtenir des résultats utiles. Par exemple, les dénombrements précis de populations pourront être remplacés par un indice d'abondance plus simple, à partir de dénombrements de crottes. Des caméras animalières fixes peuvent fournir des informations utiles sur la présence et la répartition des espèces (O'Brien, 2014). Un manuel en ligne est disponible pour le Suivi de la faune à l'aide de pièges photographiques (Ancrenaz et al., 2012). L'étendue spatiale d'une perturbation peut être estimée à l'aide d'un système de positionnement global (GPS) au sol, plutôt qu’à partir d'un avion. 
Tableau 21.2 Exemple de modèle de sélection des mesures de suivi de l'intégrité écologique

\begin{tabular}{|c|c|c|}
\hline Biodiversité & Fonctions écosystémiques & Facteurs de stress \\
\hline $\begin{array}{l}\text { Listes d'espèces } \\
\text { - } \quad \text { variation de la richesse spécifique ; } \\
\text { - nombre et étendue des espèces } \\
\text { exotiques. } \\
\text { Dynamique des populations } \\
\text { - taux de mortalité/natalité des } \\
\text { espèces indicatrices; } \\
\text { - taux d'immigration/ } \\
\quad \text { émigration des espèces } \\
\text { indicatrices ; } \\
\text { - viabilité des populations des } \\
\text { espèces indicatrices. } \\
\text { Structure trophique } \\
\text { - répartition des classes de taille de } \\
\text { - la faune : } \\
\text { niveaux de prédation. }\end{array}$ & $\begin{array}{l}\text { Succession/rétrogression } \\
\text { - } \quad \text { fréquence et ampleur des } \\
\text { perturbations (feu, insectes, } \\
\text { inondation) ; } \\
\text { - } \quad \text { répartition des classes d'âge de } \\
\text { la végétation. } \\
\text { Productivité } \\
\text { - à distance ou par site. } \\
\text { Décomposition } \\
\text { - par site. } \\
\text { Rétention des nutriments } \\
\text { - calcium et azote par site ou } \\
\quad \text { bassin versant. }\end{array}$ & $\begin{array}{l}\text { Schémas d'aménagement du } \\
\text { territoire } \\
\text { - cartes d'utilisation des terres, } \\
\text { densités routières, densités de } \\
\text { population. } \\
\text { Fragmentation des habitats } \\
\text { - taille de la parcelle, distance } \\
\text { entre parcelles, intérieur de la } \\
\text { forêt. } \\
\text { Polluants } \\
\text { - eaux usées, produits } \\
\text { pétrochimiques, etc. ; } \\
\text { transport à longue distance de } \\
\text { toxines. } \\
\text { Climat } \\
\text { - données météorologiques; } \\
\text { - fréquence des phénomènes } \\
\text { extrêmes. } \\
\text { Autres } \\
\text { - questions propres au parc. }\end{array}$ \\
\hline
\end{tabular}

Source : Woodley (1993)

\section{Suivi de l'efficacité}

Pour les aires protégées disposant de programmes actifs de gestion et de restauration, il est important de vérifier si les objectifs écologiques des mesures de gestion ont été atteints. Il est généralement facile de choisir ce qu'il faut mesurer, car les efforts sont généralement axés sur certaines espèces ou certains types d'habitats, et sur les tendances souhaitées, par exemple, des espèces autochtones plus abondantes, des espèces envahissantes moins abondantes, ou un niveau de perturbations semblable à celui de zones à faible densité humaine.

\section{Qui peut observer?}

Qui peut réellement faire un suivi écologique n'est pas une question facile. Fondamentalement, le suivi écologique est une activité scientifique. Les programmes de suivi devraient idéalement être conçus par des personnes possédant une formation scientifique, correctement testés sur le terrain et évalués par des pairs. Une fois qu'une méthode claire ou un protocole de suivi est conçu, cependant, beaucoup de personnes peuvent être formées pour recueillir des informations de suivi. Par exemple, les rangers et gardes forestiers sont des candidats idéaux, parce qu'ils patrouillent et observent régulièrement une grande partie des aires protégées. De plus en plus, des scientifiques citoyens sont formés pour fournir des données de suivi, y compris en utilisant des appareils tels que les téléphones intelligents.

La possibilité d'inclure les visiteurs et les populations autochtones et locales dans le suivi des aires protégées devrait être sérieusement envisagée. Faire participer ces personnes, et respecter leurs idées sera plus utile à une aire protégée que la simple préparation d'informations standard pour les visiteurs. Souvent, ce sont les mêmes personnes qui devront être convaincues de la nécessité d'une action dans l'aire protégée. L'inclusion des personnes tôt dans le processus renforce la confiance et la compréhension. Les sentiments et la signification spirituelle attachés aux observations par les visiteurs et les populations autochtones et locales sont essentiels, bien qu'il puisse être difficile d'inclure ces observations dans un cadre commun avec ceux du personnel et des scientifiques invités (étude de cas 21.9).

Un programme de suivi doit être conçu en fonction des besoins et de la situation particulière de chaque aire protégée. Dans de nombreux cas, des scientifiques seront à proximité, dans des universités, des agences gouvernementales ou des organisations non gouvernementales (ONG), et pourront être intéressés à mener des études de suivi à long terme. Dans d'autres cas, une aire protégée disposera d'un personnel formé. Dans de nombreuses situations, cependant, et en particulier lorsque les aires protégées manquent de personnel de recherche, les citoyens locaux et gestionnaires traditionnels des terres peuvent fournir une compréhension supplémentaire utile des écosystèmes. Peut-être la meilleure façon de penser à qui devrait participer au suivi est d'envisager le programme comme un partenariat, susceptible d'évoluer au fil du temps. Le défi, pour un gestionnaire d'aire protégée, est de s'assurer que suffisamment de personnes, disposant de suffisamment de formation, seront en mesure de rendre compte des conditions écologiques. 


\section{Étude de cas 21.9 Suivi utilisant les citoyens et les connaissances traditionnelles}

Project Noah (2014) est un site web innovant, qui enregistre l'emplacement et la date des photographies de faune prises par des scientifiques citoyens. Les photographies sont prises par des personnes intéressées et un ensemble d'instructions permet de télécharger les images d'un certain type. Par exemple, la section Oiseaux d'Afrique Sub-Saharienne compte 101 participants et plus de 1500 observations d'oiseaux. II est facile de devenir membre et de télécharger des images à partir d'un téléphone intelligent. Les photos peuvent se limiter à un weekend de bioblitz dans une aire protégée, ou être axées sur des sujets tels que la pollinisation, la phénologie ou les espèces envahissantes.

\section{Qaujimajatuqangit Inuit, Canada}

Le Qaujimajatuqangit Inuit, ou QI, représente la sagesse durement gagnée des peuples autochtones du territoire du
Nunavut, au Canada, survivants dans les rudes paysages nordiques. Cette connaissance écologique locale est un élément clé de la gouvernance locale, en particulier dans la gestion des ressources naturelles. Gilchrist et al. (2005) ont analysé l'efficacité du QI, en particulier en ce qui concerne les tendances récentes en matière de population et de répartition de quatre espèces d'oiseaux migrateurs. Pour deux des espèces évaluées, les connaissances locales ont identifié des changements de population auparavant inconnus de la science occidentale. En général, le degré de contact avec l'espèce a été un facteur important dans la détermination de la qualité des observations. Dans un cas, la répartition de l'espèce était mal comprise par les chasseurs locaux malgré les captures saisonnières. Ainsi, comme pour toute source d'information, une évaluation de la fiabilité est nécessaire.

\section{Protocoles de suivi}

Le suivi de toute entité écologique nécessite l'élaboration d'un protocole de suivi, c'est à dire un ensemble de conditions écrites spécifiant le comment, le quoi, le quand, le où et le pourquoi du suivi. Il comprend les éléments suivants :

1. Quelle est la question de suivi posée ? Par exemple, quelle est la population de grues dans l'aire protégée et cette population change-t-elle?

2. Quelle est la variable écologique à mesurer et quel est son rapport avec la question de suivi ? Par exemple, une façon utile de compter les grues pourrait être d'effectuer des comptages de printemps, lorsque les oiseaux arrivent pour la reproduction, car ils sont plus faciles à observer et à compter.

3. Quelle certitude sera nécessaire pour détecter un changement ? Il s'agit à la fois d'une question de gestion et d'une question statistique. Par exemple, si les grues sont comptées pendant deux jours, chaque printemps, il sera seulement possible de connaitre la population avec une variation de plus ou moins $20 \%$. Par conséquent, avec cette fréquence d'échantillonnage, legestionnaire nesera pasen mesure de détecter les changements d'une année à l'autre, à moins que ceux-ci ne dépassent $20 \%$. Toutefois, si la grue est une espèce menacée, le gestionnaire voudra peut-être savoir si la population change avec une certitude supérieure à $20 \%$. Cette analyse de la capacité à détecter le changement est appelé " analyse de puissance ». Des guides sont disponibles à ce sujet dans la plupart des textes statistiques en ligne (par exemple, Ellis, 2010), ou auprès d'un statisticien. Il y aura presque toujours un compromis entre le niveau de certitude dans la détection des changements et le coût d'un programme de suivi.

\section{Méthodologies de terrain}

Un ensemble clair de méthodologies permettant de détecter les changements devra être décrit. Suivant l'exemple de la grue, les méthodologies devront spécifier tous les détails requis pour un comptage printanier, y compris où aller, quand compter, s'il faut compter les juvéniles séparément des adultes, etc. Cette section devra être très spécifique, afin que les méthodologies puissent être facilement répétées par différents observateurs.

\section{Collecte et stockage des données}

Cette partie d'un protocole se réfere à comment les données seront collectées, comment les données seront stockées et quels contrôles de qualité sont nécessaires. Par exemple, une feuille de données de terrain pour le comptage des grues peut être préparée, incluant toutes les métadonnées (nom de l'observateur, date, lieu, etc.), ainsi que les données de comptage réelles. La feuille de données ira ensuite dans un fichier de stockage (qui pourra être dupliqué à des fins de sauvegarde), et ses données pourront être saisies dans une feuille de calcul informatique ou une base de données. Un bon protocole devra inclure des règles de contrôle de qualité, afin de s'assurer que les observations soient transférées correctement de la feuille de travail de terrain à l'ordinateur. Le contrôle de la qualité peut également inclure une personne indépendante vérifiant les chiffres.

\section{Analyse des données}

Un protocole devra préciser comment un ensemble de mesures sera analysé, y compris les méthodes statistiques et les moyens de déterminer l'importance d'une observation. Pour l'exemple des grues, si les données sur 10 ans montraient, à un niveau de confiance de $95 \%$, que les populations de grues subissent une baisse de $2 \%$ par an, cela entraînerait-il une mesure de gestion ? 


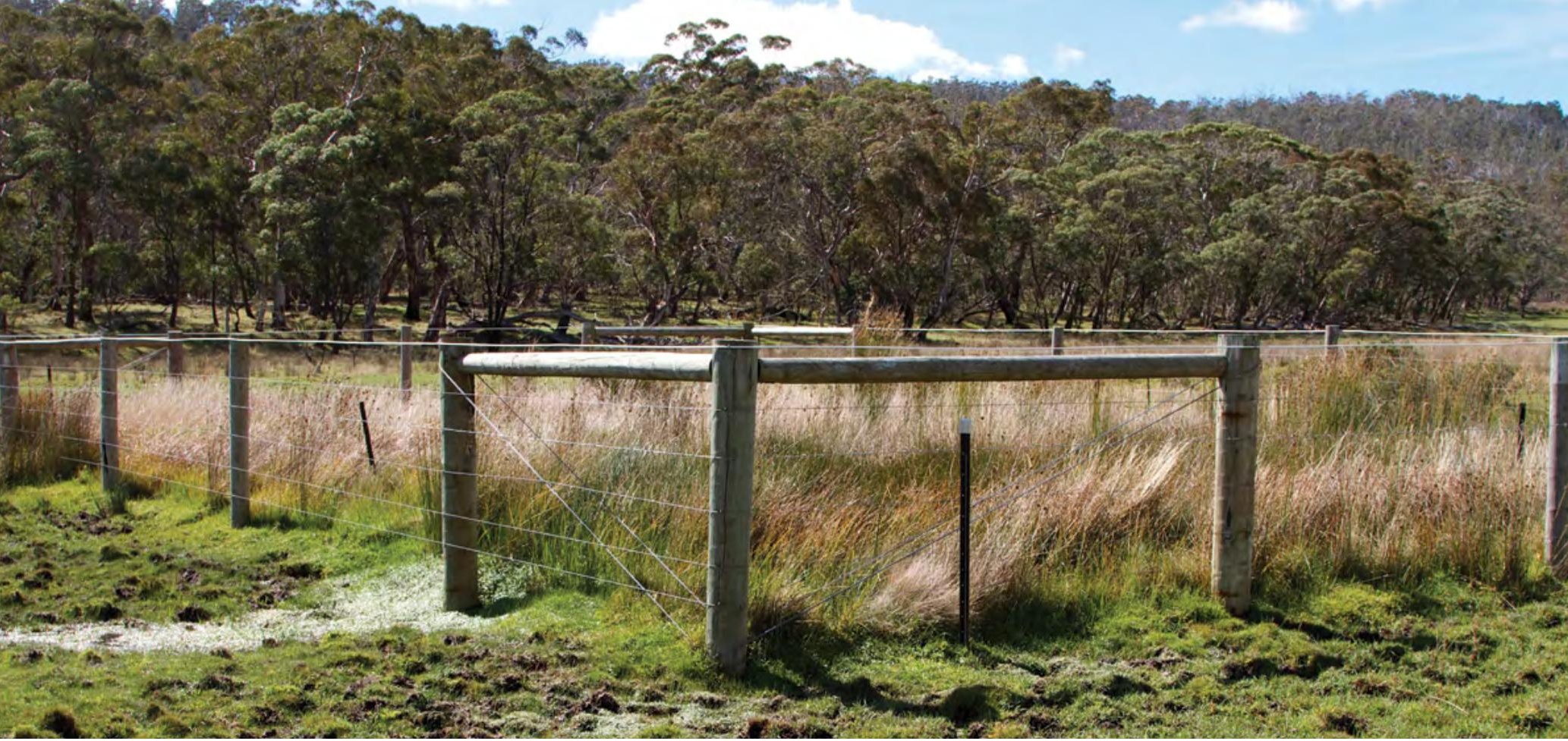

Les gestionnaires de parc ont souvent recours à des enclos de suivi à long terme pour déterminer les répercussions de l'introduction d'espèces telles que les chevaux sauvages dans le parc national alpin du Victoria, Alpes australiennes

Source : lan Pulsford

4. Le résultat est-il affecté par des interactions avec d'autres espèces ou processus?

Pour répondre à ces questions, les gestionnaires ont besoin d'une conception du suivi. Tout programme de suivi partira d'un certain nombre d'hypothèses au sujet de la zone de réserve affectée, des changements susceptibles d'être détectés et des niveaux de certitude. Un statisticien ou scientifique peut aider à définir ces hypothèses et à renforcer la conception. Le tableau 21.3 fournit des indications sur le nombre minimal d'observations nécessaires pour répondre à certaines questions.

Le suivi n'est utile que si les résultats sont analysés et évalués, et intégrés dans des mesures de gestion du suivi. Le tableau 21.3 recommande la taille minimale des échantillons pour détecter des différences assez évidentes dans un écosystème. Chaque exemple dans le tableau suppose une probabilité de $20 \%$ de résultats faux-positifs et une probabilité de $20 \%$ de résultats faux-négatifs.

L'analyse des données pour répondre à la question "les mesures de gestion ont-elles été efficaces?» est généralement plus facile à aborder que le suivi des conditions. Au lieu de se demander ce qu'un écosystème "devrait " faire, le gestionnaire se demande s'il a fait ce qui était prévu, suite à une intervention de gestion spécifique. Il est souvent important de limiter la question de l'efficacité au calendrier de la gestion pratique, indépendamment de la durée de vie des espèces concernées ou de la rapidité des processus, car le financement de projets de gestion des aires protégées se fait généralement à court terme, et nécessite donc des mesures de résussite à court terme. Étant donné que les écosystèmes présenteront des retards de réponse, le suivi de l'efficacité devra toutefois comporter des mesures à plus long terme, liées au programme de suivi des conditions de l'aire protégée.

Les cibles utilisées pour le suivi de l'efficacité peuvent inclure des attentes claires quant aux impacts, comme le pourcentage de superficie traitée avec succès ou la taille de population d'une espèce obtenue grâce à la gestion. Ces objectifs devront représenter une réponse de la part de l'écosystème, plutôt qu'une mesure de l'effort appliqué. L'atteinte de ces objectifs exige généralement moins d'attention aux hypothèses statistiques, si importantes dans le suivi des conditions.

\section{Registre à long terme des observations de suivi}

L'information de suivi devra être stockée dans un endroit facilement accessible, sûr à long terme, et bien documentée. La plupart des organisations d'aires protégées pourraient améliorer la gestion de leurs données. De nombreuses observations sont perdues, à long terme, ou ne disposent pas de métadonnées adéquates. Des normes formelles de métadonnées sont disponibles, décrivant l'information devant accompagner les données collectées, y compris la façon dont elles ont été collectées, qui les a collecté, les méthodes exactes utilisées, les emplacements, etc. Les métadonnées sont aussi importantes que la donnée ellemême. Pour les données sur la biodiversité, le " Darwin Core ", disponible en ligne, constitue une norme commune de métadonnées (Wieczorek et al., 2012). Le Darwin Core définit simplement une liste d'informations devant être inclues dans les métadonnées, telles que la date, le nom de 
l'espèce ou les coordonnées géographiques. Beaucoup trop de données sont inexploitables, simplement parce qu'elles manquent des métadonnées.

Les observations répétées des gestionnaires d'aires protégées et des peuples autochtones peuvent se traduire par des normes de gestion des données renforçant la compréhension de l'aire protégée au fil du temps. Les clés du suivi incluent : 1) des protocoles de suivi clairs, conservant une même technique de mesure cohérente dans le temps, et entre les sites et les observateurs; et 2) un stockage de données accessible offrant des observations de domaine public. Il est important de trouver un référentiel approprié (encadré 21.5).

La publication des données de suivi et de leur protocole n'est que le début de la conservation des données à long terme. Afin d'assurer l'accessibilité à long terme (50-100 ans) des données de suivi, il faudra être conscient des changements affectant les médias électroniques. L'information électronique, comme les fichiers papier, doit être organisée. Elle doit faire face au risque supplémentaire d'être éliminée par des procédures de sauvegarde défectueuses. La meilleure garantie que les données de suivi survivent est de s'assurer qu'elles soient régulièrement utilisées et mises à jour par les organisations de conservation.

\section{Conclusion}

La gestion des aires protégées est un travail de plus en plus complexe, qui exige une bonne compréhension de l'écologie du site, ainsi que de quelques éléments fondamentaux sur le fonctionnement des écosystèmes. Vous trouverez ci-dessous un résumé des messages clés de ce chapitre à l'intention des gestionnaires d'aires protégées.

1. Les aires protégées, en tant qu'écosystèmes, se caractérisent à la fois par une biodiversité et des fonctions écosystémiques de soutien. Les deux sont connectées et s'influencent mutuellement. Il n'est pas possible de mettre en place une gestion uniquement pour une espèce ou un type d'écosystème, sans également tenir compte des processus écologiques qui les soutiennent.

2. Le suivi des conditions des écosystèmes et des mesures de gestion est un élément fondamental de la gestion des aires protégées. Les systèmes de suivi doivent faire partie du cadre global de gestion d'une aire protégée. Un système de suivi doit reposer sur une compréhension fondamentale de la structure et de la fonction des écosystèmes.

3. La science appliquée de la biologie de la conservation fournit une gamme d'outils et d'approches bien

\section{Encadré 21.5 Trouver un référentiel pour stocker les informations de suivi}

Denombreuxgouvernements ou ONG disposent peut-être déjà d'un catalogue de données ouvert, où les données peuvent être publiées (par exemple, <datacatalogs.org>). Un site web interne peut également permettre de publier des informations à l'intention du personnel.

Un certain nombre de référentiels spécifiques sont également disponibles pour les aires protégées et la conservation. Le Global Biodiversity Information Facility (GBIF / Système mondial d'information sur la biodiversité) est une infrastructure internationale de données ouvertes pour les informations sur la biodiversité. II permet à n'importe qui, n'importe où, d'accéder à des données sur tous les types de vie sur Terre, partagées au-delà des frontières nationales via internet.

Protected Planet (Planète protégée) est un site web dynamique (<www.protectedplanet.net $>$ ) hébergé par le PNUE-WCMC, et cherchant à décrire les aires protégées du monde. L'Open Parks Network (Réseau ouvert des parcs) est un pipeline de connaissances pour les professionnels des parcs, hébergeant des archives numérisées et des informations difficiles à trouver (OPM, 2014).

développés pour la gestion de la biodiversité à toutes les échelles, du niveau génétique à la communauté écologique. Les approches de gestion des populations sont les mieux développées, et incluent des considérations détaillées pour la gestion de la diversité génétique, des métapopulations et de la viabilité.

4. Une gestion des fonctions écologiques est possible, et nécessaire, dans de nombreuses aires protégées. L'utilisation du feu, dans des incendie adaptés aux écosystèmes, en est un bon exemple.

5. Une grande partie de la gestion des aires protégées se concentre sur la gestion des risques écologiques. Ce chapitre présente une approche formelle et structurée pour définir, évaluer et classer les menaces écologiques dans les aires protégées.

La science soutenant l'application de la gestion à la biodiversité et à la fonction des écosystèmes est vaste et croissante. Ce chapitre vise à donner un aperçu des principes et perspectives clés, mais ne peut pas couvrir tous les domaines. Les gestionnaires d'aires protégées pourront tirer parti de l'expertise bénévole au sein des Commissions de l'UICN, y compris la Commission mondiale des aires protégées, pour les aider à résoudre les problèmes de gestion des aires protégées. 


\section{Références}

Lectures recommandées

Dd Akçakaya, H. R. et Sjögren-Gulve, P. (2000) 'Population viability analyses in conservation planning: an overview', Ecological Bulletins 9: 9-21.

Ancrenaz, M., Hearn, A. J., Ross, J., Sollmann, R. et Wilting, A. (2012) Handbook for Wildlife Monitoring Using Camera Traps, BBEC II Secretariat, Natural Resources Office, Chief Minister's Department, Sabah, Malaisie.

Ashton, D. H. (1981) 'Fire in tall open forests (wet sclerophyll forests)', in A. M. Gill, R. H. Groves and I. R. Noble (eds) Fire and the Australian Biota, pp. 339-66, Australian Academy of Science, Canberra.

Baker, W. 1. (1995) 'Long-term response of disturbance landscapes to human intervention and global change', Landscape Ecology 10: 143-59.

Balmford, A., Carey, P., Kapos, V., Manica, A., Rodrigues, A. S. L., Scharlemann, J. P. W. et Green, R. E. (2009) 'Capturing the many dimensions of threat', Conservation Biology 23: 482-7.

Beissinger, S. R. (2002) 'Population viability analysis: past, present, future', in S. R. Beissinger and D. R. McCullough (eds) Population Viability Analysis, pp. 5-17, University of Chicago Press, Chicago.

Beissinger, S. R. et McCullough, D. R. (eds) (2002) Population Viability Analysis, University of Chicago Press, Chicago.

Benz, C. (1996) 'Evaluating attempts to reintroduce sea otters along the California coastline', Endangered Species Update 13: 31-5.

Bouzat, J. L., Cheng, H. H., Lewin, H. A., Westemeier, L., Brawn, J. D. et Paige, K. N. (1998) 'Genetic evaluation of a demographic bottleneck in the greater prairie chicken', Conservation Biology 12: 836-43.

Bouzat, J. L., Johnson, J. A., Toepfer, J. E., Simpson,A., Esker, T. L. et Westemeier, R. L. (2009) 'Beyond the beneficial effects of translocations as an effective tool for the genetic restoration of isolated populations', Conservation Genetics 10: 191-201.

Boyce, M. S. et Daley, D. J. (1980) 'Population tracking of fluctuating environments and natural selection for tracking ability', American Naturalist 115(4): 480-91.
Bruner, A. G., Gullison, R. E., Rice, R. E. et Fonseca, G. A. B. (2001) 'Effectiveness of parks in protecting tropical biodiversity', Science 291: 25-128.

Burns, C. E., Johnston, K. M. et Schmitz, O. J. (2003) 'Global climate change and mammalian species diversity in US national parks', Proceedings of the National Academy of Sciences 100: 11474-7.

Butchart, S. H. M. et 44 others (2010) 'Global biodiversity: indicators of recent declines', Science 328: 1164-8.

Cairns, J. (1977) 'Quantification of biological integrity', in R. K. Ballentine and L. J. Guarraia (eds) The Integrity of Water, US EPA Office of Water and Hazardous Materials, US Government Printing Office, Washington, DC.

Campbell, A., Kapos, V., Lysenko, I., Scharlemann, J. P. W., Dickson, B., Gibbs, H. K., Hansen, M. et Miles, L. (2008) Carbon Emissions from Forest Loss in Protected Areas, UNEP-WCMC, Cambridge.

Cardinale, B. J., Duffy, J. E., Gonzalez, A., Hooper, D. U., Perrings, C., Venail, P., Narwani, A., Mace, G. M., Tilman, D., Wardle, D. A., Kinzig, A. P., Daily, G. C., Loreau, M., Grace, J. B., Larigauderie, A., Srivastava, D. S. et Naeem, S. (2012) 'Biodiversity loss and its impact on humanity', Nature 486: 59-67.

Carvalheiro, L. G., Seymour, C. L., Veldtman, R. et Nicolson, S. W. (2010) 'Pollination services decline with distance from natural habitat even in biodiversityrich areas', Journal of Applied Ecology 47: 810-20.

Caughley, G. (1994) 'Directions in conservation biology', Journal of Animal Ecology 63: 215-44.

Chan, K. M., Shaw, M. R., Cameron, D. R., Underwood, E. C. et Daily, G. C. (2006) 'Conservation planning for ecosystem services’, PLoS Biology 4(11): e379.

Chapman, C. A., Lawes, M. J., Naughton-Treves, L. et Gillespie, T. (2003) 'Primate survival in communityowned forest fragments: are metapopulation models useful amidst intensive use?', in L. K. Marsh (ed.) Primates in Fragments: Ecology and conservation, pp. 63-78, Kluwer Academic/Plenum Publishers, New York.

Clark, S., Bolt, K. et Campbell, A. (2008) Protected areas: an effective tool to reduce emissions from deforestation and forest degradation in developing countries, Working Paper, UNEP-WCMC, Cambridge. 
Clevenger, A. P. et Sawaya, M. A. (2010) 'Piloting a non-invasive genetic sampling method for evaluating population-level benefits of wildlife crossing structures', Ecology and Society 15: 7.

Clevenger, A. P., Ford, A. T. et Sawaya, M. A. (2009) Banff wildlife crossings project: integrating science and education in restoring population connectivity across transportation corridors, Final report to Parks Canada Agency, Radium Hot Springs, Colombie-Britannique.

Conservation Measures Partnership (CMP) (2005) Taxonomies of Direct Threats and Conservation Actions, Conservation Measures Partnership, Washington, DC.

DDd Conservation Measures Partnership (CMP) (2013) The Open Standards for the Practice of Conservation, Conservation Measures Partnership, Washington, DC. $<$ www.conservationmeasures.org>

Convention on Biological Diversity (CBD) (2004) Programme of Work on Protected Areas, Secretariat of the Convention on Biological Diversity, Montréal. $<$ www.cbd.int/programmes/pa/pow-goals-alone. pdfs

Corlatti, L., Hacklaender, K. et Frey-Roos, F. (2009) 'Ability of wildlife overpasses to provide connectivity and prevent genetic isolation', Conservation Biology 23: $548-56$.

Daily, C. (ed.) (1997) Naturés Services: Societal dependence on natural ecosystems, Island Press, Washington, DC.

Deguise, I. E. et Kerr, J. (2006) 'Protected areas and prospects for endangered species conservation in Canada', Conservation Biology 20(1): 48-55.

di Minin, E., Hunter, L. T., Balme, G. A., Smith, R. J., Goodman, P. S. et Slotow, R. (2013) 'Creating larger and better connected protected areas enhances the persistence of big game species in the MaputalandPondoland-Albany biodiversity hotspot', PloS ONE 8: e71788.

Dd Dudley, N. (ed.) (2008) Guidelines for Applying Protected Area Management Categories, CMAP-UICN, Gland.

Dufrene, M. et Legendre, P. (1977) 'Species assemblages and indicator species: the need for a flexible asymmetrical approach', Ecological Monographs 67: $345-66$.

Duggins, D. O. (1980) 'Kelp beds and sea otters: an experimental approach', Ecology 61: 447-53.
Edwards, C. J. et Regier, H. A. (eds) (1990) An Ecosystem Approach to the Integrity of the Great Lakes in Turbulent Times, Special Publication 90-4, Great Lakes Fishery Commission, Ann Arbor, MI.

Ellis, P. D. (2010) The Essential Guide to Effect Sizes: An introduction to statistical power, meta-analysis and the interpretation of research results, Cambridge University Press, Cambridge.

DDl Ervin, J. (2002) WWF Rapid Assessment and Prioritization of Protected Area Management (RAPPAM) Methodology, WWF, Gland.

Estes, J. A., Terborgh, J., Brashares, J. S., Power, M. E., Berger, J., Bond, W. J. et Wardle, D. A. (2011) 'Trophic downgrading of planet Earth', Science 333(6040): 301-6.

Fagan, W. F. et Holmes, E. E. (2006) 'Quantifying the extinction vortex', Ecology Letters 9(1): 51-60.

Ferraro, K. P. J., Pfaff, A., Sanchez-Azofeifa, A. et Robalino, J. (2008) 'Measuring the effectiveness of protected area networks in reducing deforestation', Proceedings of the National Academy of Science 105(41).

Firecast (2014) Firecast Forest and Fire Monitoring and Alert System for the Tropics. <firealerts.conservation. org>

Flather, C. H., Hayward, G. D., Beissinger, S. R. et Stephens, P. A. (2011) 'Minimum viable populations: is there a "magic number" for conservation practitioners?', Trends in Ecology and Evolution 26(6): $307-16$.

Fortin, D., Beyer, H. L., Boyce, M. S., Smith, D. W., Duchesne, T. et Mao, J. S. (2005) 'Wolves influence elk movements: behavior shapes a trophic cascade in Yellowstone National Park', Ecology 86: 1320-30.

Foundations of Success (FOS) (2014) Foundations of Success. <www.fosonline.org/>

Frankham, R. (2005) 'Genetics and extinction', Biological Conservation 126: 131-40.

Freedman, B. et Hutchinson, T. C. (1980) 'Long term effects of smelter pollution at Sudbury, Ontario, on surrounding forest communities', Canadian Journal of Botany 58: 2123-40.

Gaston, K. J. (2000) 'Global patterns in biodiversity', Nature 405: 220-7. 
Dd Geldmann, J., Barnes, M., Coad, L., Craigie, I. D., Hockings, M. et Burgess, N. D. (2013) 'Effectiveness of terrestrial protected areas in reducing habitat loss and population declines', Biological Conservation 161: $230-8$.

Gilchrist, G., Mallory, M. et Merkel, F. (2005) 'Can local ecological knowledge contribute to wildlife management? Case studies of migratory birds', Ecology and Society 10: 20.

Gilpin, M. E. et Soulé, M. E. (1986) Conservation Biology: The science of scarcity and diversity, Sinauer Associates, Sunderland, MA.

Gitzen, R. A., Millspaugh, J. J., Cooper, A. B. et Licht, D. S. (eds) (2012) Design and Analysis of Long-Term Ecological Monitoring Studies, Cambridge University Press, New York.

Gurd, D. B., Nudds, T. D. et Rivard, D. H. (2001)

'Conservation of mammals in eastern North American wildlife reserves: how small is too small?', Conservation Biology 15: 1355-63.

Hall, J. A. et Fleischman, E. (2010) 'Demonstration as a means to translate conservation science into practice', Conservation Biology 24: 120-7.

Hansen, M. C., Stehman, S. V., Potapov, P. V., Loveland, T. R., Townshend, J. R. G., de Fries, R. S., Pittman, K. W., Arunarwati, B., Stolle, F., Steininger, M. K., Carroll, M. et di Miceli, C. (2008) 'Humid tropical forest clearing from 2000 to 2005 quantified by using multitemporal and multiresolution remotely sensed data', Proceedings of the National Academy of Sciences. <doi:10.1073/ pnas.0804042105>

Hanski, I. et Simberloff, D. (1997) 'The metapopulation approach, its history, conceptual domain, and application to conservation', in I. Hanski et M. E. Gilpin (eds) Metapopulation Biology: Ecology, genetics, and evolution, pp. 5-26, Academic Press, San Diego.

Hebblewhite, M., White, C. A., Nietvelt, C. G., McKenzie, J., Hurd, T. E., Fryxell, J. M., Bayley, S. E. et Paquet, P. (2005) 'Human activity mediates a trophic cascade caused by wolves', Ecology 86: 213544.

Heywood, V. H. (ed.) (1995) Global Biodiversity Assessment, Cambridge University Press, Cambridge.
Hooper, D. U., Adair, E. C., Cardinale, B. J., Byrnes, J. E. K., Hungate, B. A., Matulich, K. L., Gonzalez, A., Duffy, J. E., Gamfeldt, L. et O'Connor, M. I. (2012) 'A global synthesis reveals biodiversity loss as a major driver of ecosystem change', Nature 486: 105-8.

Hughes, A. R., Inouye, B. D., Johnson, M. T., Underwood, N. et Vellend, M. (2008) 'Ecological consequences of genetic diversity', Ecology Letters 11 : 609-23.

International Joint Commission (1978) Revised Great Lakes Water Quality Agreement of 1978, International Joint Commission, États-Unis d'Amérique et Canada.

International Union for Conservation of Nature (IUCN) (2001) IUCN Red List Categories and Criteria. Version 3.1, Species Survival Commission, UICN, Gland.

International Union for Conservation of Nature (IUCN) (2005a) Conservation Actions Authority File. Version 1.0, IUCN Species Survival Commission, Cambridge. <iucn.org/webfiles/doc/SSC/RedList/ AuthorityF/ consactions.rtf>

International Union for Conservation of Nature (IUCN) (2005b) Threats Authority File. Version 2.1, IUCN Species Survival Commission, Cambridge. <iucn.org/ webfiles/doc/SSC/RedList/AuthorityF/ threats.rtf>

International Union for Conservation of Nature (IUCN) (2006) Habitats Authority File. Version 1.0, IUCN Species Survival Commission, Cambridge. <iucn.org/ webfiles/doc/SSC/RedList/AuthorityF/ habitats.rtf>

Janzen, D. (1999) 'Gardenification of tropical conserved wildlands: multitasking, multicropping and multiusers', Proceedings of the National Academy of Sciences 96: 5987-94.

Klijn, F. (1994) 'Ecosystem classification for environmental management', Ecology and Environment 2: $85-116$.

Klijn, F. et de Haes, H. A. U. (1994) A Hierarchical Approach to Ecosystems and its Implications for Ecological Land Classification, Springer, Berlin.

Landres, P. B., Verner, J. et Thomas, J. W. (1988) 'Ecological uses of vertebrate indicator species', Conservation Biology 2: 316-28.

Landry, M., Thomas, V. G. et Nudds, T. D. (2001) 'Sizes of Canadian national parks and the viability of large mammal populations: policy implications', George Wright Forum 18(1): 13-23. 
Le Saout, S., Hoffmann, M., Shi, Y., Hughes, A., Bernard, C., Brooks, T. M., Bertzky, B., Butchart, S. H. M., Stuart, S. N., Badman, T. et Rodrigues, A. S. L. (2013) 'Protected areas and effective biodiversity conservation', Science 15: 803-5.

Levins, R. (1970) 'Extinction', Lectures on Mathematics in the Life Sciences 2: 75-107.

Likens, G. E., Bormann, F. H., Pierce, R. S. et Reiners, W. A. (1978) 'Recovery of a deforested ecosystem', Science 199: 492-6.

Lindenmayer, D. B. (2009) Forest Pattern and Ecological Process: A synthesis of 25 years of research, CSIRO Publishing, Melbourne.

Lindenmayer, D. B. et Franklin, J. F. (2002) Conserving Forest Biodiversity: A comprehensive multiscaled approach, Island Press, Washington, DC.

Lindenmayer, D. B. et Lichens, G. F. (2010) Effective Ecological Monitoring, CSIRO Publishing, Melbourne.

Lindenmayer, D. B., Hobbs, R. J., Likens, G. E., Krebs, C. et Banks, S. C. (2011) 'Newly discovered landscape traps produce regime shifts in wet forests', Proceedings of the National Academy of Sciences 108: 15 887-91.

Lindenmayer, D. B., Mackey, B. G., Mullen, I. C., McCarthy, M. A., Gill, A. M., Cunningham, R. B. et Donnelly, C. F. (1999) 'Factors affecting stand structure in forests - are there climatic and topographic determinants?', Forest Ecology and Management 123: 55-63.

McCullough, D. R. (ed.) (1996) Metapopulations and Wildlife Conservation, Island Press, Washington, DC. MacKinnon, J., MacKinnon, K., Child, G. et Thorsell, J. (1986) Managing Protected Areas in the Tropics, UICN et PNUE, Cambridge.

MacKinnon, K. (2005) 'Parks, peoples and policies: conflicting agendas for forests in Southeast Asia', in E. Bermingham, C. W. Dick et C. Moritz (eds) Tropical Rainforests: Past, present and future, pp. 558-82, University of Chicago Press, Chicago.

MacKinnon, K., Hatta, G., Halim, H. et Mangalik, A. (1996) The Ecology of Kalimantan: Indonesian Borneo, Periplus, Singapour.

MacKinnon, J., MacKinnon, K., Child, G. et Thorsell, J. (1986) Managing Protected Areas in the Tropics, UICN, Gland.
Margoluis, R. et Salafsky, N. (1998) Measures of Success: Designing, managing, and monitoring conservation and development projects, Island Press, Washington, DC.

Margules, C. R. et Pressey, R. L. (2000) 'Systematic conservation planning', Nature 405: 243-53.

Martin, L. (1990) 'Flying foxes of the Brisbane River', in P. Davie, E. Stock et D. Low Choy (eds) The Brisbane River: A source-book for the future, Australian Littoral Society in association with the Queensland Museum, Brisbane.

Mattila, H. R. et Seeley, T. D. (2007) 'Genetic diversity in honey bee colonies enhances productivity and fitness', Science 317(5836): 362-4. Mills, L. S., Soulé, M. E. et Doak, D. F. (1993) 'The keystone-species concept in ecology and conservation', BioScience 43(4): 219-24.

Dd Miradi (2007) Miradi Adaptive Management Software for Conservation Projects. <www.miradi.org>

Murphy, C. et Robertson, A. W. (2000) Preliminary Study of the Effects of Honey Bees (Apis mellifera) in Tongariro National Park, New Zealand Department of Conservation, Wellington.

National Parks Service (NPS) (2006) US National Parks Service Management Policies: The guide to managing the national park system, US National Parks Service, Washington, DC. <www.nps.gov/policy/mp/ chapter4.htm>

National Parks Service (NPS) (2014) US National Parks Service Inventory and Monitoring Program, US National Parks Service, Washington, DC. <science. nature.nps. gov/im/>

Nel, P. (1997) Conservation and Management of Biodiversity in Protected Areas in the North West Province, Conservation Management Division, North West Parks and Tourism Board, Mafikeng, Afrique du Sud. <www.cbd.int/doc/meetings/nbsap/nbsapcbwseafr-01/other/nbsapcbw-seafr-01-nwptb- pa-en.pdf>

Newmark, W. D. (1995) 'Extinction of mammal populations in western North American national parks', Conservation Biology 9: 512-26.

Niemi, G. J. et McDonald, M. E. (2004) 'Application of ecological indicators', Annual Review of Ecology, Evolution and Systematics 35: 89-111.

Noss, R. F. (1983) 'A regional landscape approach to maintain diversity', BioScience 33(11): 700-6. 
Noss, R. F. (1990) 'Indicators for monitoring biodiversity: a hierarchical approach', Conservation Biology 4: 355-64.

Oakley, K. L., Thomas, L. P. et Fancy, S. G. (2003) 'Guidelines for long-term monitoring protocols', Wildlife Society Bulletin 31: 1000-3.

O'Brien, T. (2014) Camera Traps for Conservation: Monitoring protected area investment, Zoological Society of London, Londres.

Ojeda, V. S., Suarez, M. L. et Kitzberger, T. (2007) 'Crown dieback events as key processes creating cavity habitat for magellanic woodpeckers', Austral Ecology 32(4): 436-45.

Ollerton, J., Winfree, R. et Tarrant, S. (2011) 'How many flowering plants are pollinated by animals?', Oikos 120: 321-6.

Dd Olson, D., Dinerstein, E., Wikramanayake, E. D., Burgess, N. D., Powell, G. V. N., Underwood, E. C., d'Amico, J. A., Itoua, I., Strand, H., Morrison, J. C., Loucks, C. J., Allnutt, T., Ricketts, T. H., Kura, Y., Lamoreux, J. F., Wettengel, W. W., Hedao, P. et Kassem, K. R. (2001) 'Terrestrial ecoregions of the world: a new map of life on earth', BioScience 51: 933-8.

Open Parks Network (OPM) (2014) Open Parks Network. <openparksnetwork.org/>

Pacioni, C., Wayne, A. D. et Spencer, P. B. S. (2011) 'Effects of habitat fragmentation on population structure and long-distance gene flow in an endangered marsupial: the woylie', Journal of Zoology 283: 98-107.

Paine, R. T. (1969) 'A note on trophic complexity and community stability', American Naturalist 103: 91-3.

Patrick, R. (1967) 'Diatom communities in estuaries', American Association for the Advancement of Science (83): 311-15.

Pechmann, H. K., Scott, D. E., Semlitsch, R. D., Caldwell, I. P., Vitt, L. et Gibbons, J. W. (1991) 'Declining amphibian populations: the problem of separating human impacts from natural fluctuations', Science 253: 892-5.

Pimentel, D., Westra, L. et Noss, R. (eds) (2000) Ecological Integrity: Integrating environment, conservation, and health, Island Press, Washington, DC.
Pimm, S. L. (1982) Food Webs, Chapman \& Hall, Londres.

Project Noah (2014) A Tool to Explore and Document Wildlife and a Platform to Harness the Power of Citizen Scientists Everywhere, Project Noah. <www. projectnoah.org>

Rabinowitz, D. (1981) 'Seven forms of rarity', in H. Synge (ed.) The Biological Aspects of Rare Plant Conservation, pp. 205-17, John Wiley \& Sons, Hoboken, NJ.

Raesly, E. J. (2001) 'Progress and status of river otter reintroduction projects in the United States', Wildlife Society Bulletin 29: 856-62.

Rafferty, C. et Pacioni, C. (2012) Woodland reserve, Whiteman Park: a case study in the conservation value of post-care animals, Australian Wildlife Rehabilitation Conference, Townsville, Queensland. <www.awrc.org. au/uploads/5/8/6/6/5866843/ rafferty_pacioni_value_ post_care_2012.pdf>

Ripple, W. J., Larsen, E. J., Renkin, R. A. et Smith, D. W. (2001) 'Trophic cascades among wolves, elk, and aspen on Yellowstone National Park's northern range', Biological Conservation 102: 227-34.

Rivard, D. H., Poitevin, J., Plasse, D., Carleton, M. et Currie, D. J. (2000) 'Changing species richness and composition in Canadian national parks', Conservation Biology 14: 1099-109.

Salafsky, N. et Margoluis, R. (1999) 'Threat reduction assessment: a practical and cost- effective approach to evaluating conservation and development projects', Conservation Biology 13: 830-41.

Salafsky, N., Butchart, S. H. M., Salzer, D., Stattersfield, A. J., Neugarten, J., Hilton-Taylor, C., Collen, B., Master, L., O'Connor, S. et Wilkie, D. (2009) 'Pragmatism and practice in classifying threats: reply to Balmford et al.', Conservation Biology 23: 488-93.

Salafsky, N., Salzer, D., Ervin, J., Boucher, T. et Ostlie, W. (2003) Conventions for Defining, Naming, Measuring, Combining, and Mapping Threats in Conservation: An initial proposal for a standard system, Conservation Measures Partnership. <www.conservationmeasures. org> 
Salafsky, N., Salzer, D., Stattersfield, A. J., Hilton-Taylor, C., Neugarten, R., Butchart, S. H. M., Collen, B., Cox, N., Master, L. L., O'Connor, S. et Wilkie, D. (2008) 'A standard lexicon for biodiversity conservation: unified classifications of threats and actions', Conservation Biology 22: 897-911.

Sawaya, M. A., Clevenger, A. P. and Kalinowski, S. T. (2013) 'Demographic connectivity for ursid populations at wildlife crossing structures in Banff National Park', Conservation Biology 27: 721-30.

Schulze, E. D. et Mooney, H. A. (1993) Biodiversity and Ecosystem Function, Springer Verlag, New York.

Shaffer, M. (1978) Determining minimum viable population sizes: a case study of the grizzly bear (Ursus arctos L.), PhD dissertation, Duke University, Durham, NC.

Simberloff, D. (1998) 'Flagships, umbrellas, and keystones: is single-species management passé in the landscape era?', Biological Conservation 83: 247-57.

Soulé, M. E. et Simberloff, D. (1986) 'What do genetics and ecology tell us about the design of nature reserves', Biological Conservation 35: 19-40.

Dd Spalding, M. D., Fox, H. E., Allen, G. R., Davidson, N., Ferdaña, Z. A., Finlayson, M., Halpern, B. S., Jorge, M. A., Lombana, A., Lourie,

S. A., Martin, K. D., McManus, E., Molnar, J., Recchia, C. A. et Robertson, J. (2007) 'Marine ecoregions of the world: a bioregionalization of coastal and shelf areas', Bioscience 57: 573-83.

Steege, H. et 119 others (2013) 'Hyperdominance in the Amazonian tree flora', Science 342: 1243092.

Stokes, M. (2012) 'Population ecology at work: managing game populations', Nature Education Knowledge 3(10): 5.

DD The Nature Conservancy (TNC) (2000) The Five-S Framework for Site Conservation: A practitioner's handbook for site conservation planning and measuring conservation success. Volume I, 2nd edn, The Nature Conservancy, Arlington, VA.

DDd The Nature Conservancy (TNC) (2007) Conservation Action Planning: Developing strategies, taking action and measuring success at any scale, The Nature Conservancy, Arlington, VA.
Tilman, D. et Kareiva, P. M. (eds) (1997) Spatial Ecology: The role of space in population dynamics and interspecific interactions. Volume 30, Princeton University Press, Princeton, NJ.

Tingley, M. W., Estes, L. D. et Wilcove, D. S. (2013) 'Climate change must not blow conservation off course', Nature 500: 271-2.

Traill, L. W., Brook, B. W., Frankham, R. R. et Bradshaw, C. J. (2010) 'Pragmatic population viability targets in a rapidly changing world', Biological Conservation 143: 28-34.

Tucker, C. J., Pinzon, J. E., Brown, M. E., Slayback, D. A., Pak, E. W., Mahoney, R., Vermote, E. F. et El Saleous, N. (2005) 'An extended AVHRR 8-km NDVI dataset compatible with MODIS and SPOT vegetation NDVI data', International Journal of Remote Sensing 26: 4485-98.

Udvardy, M. D. F. (1975) A classification of the biogeographical provinces of the world, IUCN Occasional Paper No. 18, UICN, Gland.

Underwood, A. J. (1994) 'On beyond BACI: sampling designs that might reliably detect environmental disturbances', Ecological Applications 4: 3-15. van der Ree, R., Heinze, D., McCarthy, M. et Mansergh, I. (2009) 'Wildlife tunnel enhances population viability', Ecology and Society 14(2). <www.ecologyandsociety. org/vol14/iss $2 /$ art $7 />$

Watson, L. H., Odendaal, H. E., Barry, T. J. et Pietersen, J. (2005) 'Population viability of Cape mountain zebra in Gamka Mountain Nature Reserve, South Africa: the influence of habitat and fire', Biological Conservation 122: $173-80$.

Westemeier, R. L., Brawn, J. D., Simpson, S. A., Esker, T. L., Jansen, R. W., Walk, J. W., Kershner, E. L., Bouzat, J. L. et Paige, K. N. (1998) 'Tracking the long-term decline and recovery of an isolated population', Science 282: 1695-8.

Westman, W. E. (1990) 'Managing for biodiversity', Bioscience 40: 26-33.

White, C. A. et Fisher, W. (2007) 'Ecological restoration in the Canadian Rocky Mountains: developing and implementing the 1997 Banff National Park Management Plan', in M. Price (ed.) Mountain Area Research and Management, pp. 217- 42, Earthscan, Londres. 
White, C. A., Hurd, T. E., Hebblewhite, M. et Pengelly, I. R. (2007) Mitigating fire suppression, highway and habitat fragmentation effects in the Bow Valley ecosystem, Banff National Park: preliminary evaluation with a before-after-control- impact (BACI) design with path analysis, Paper presented at Monitoring the Effectiveness of Biological Conservation Conference, Richmond, Colombie-Britannique, 2-4 novembre 2004. <www.forrex.org/events/mebc/papers.html>

White, C. A., Olmsted, C. E. et Kay, C. E. (1998) 'Aspen, elk, and fire in the Rocky Mountain national parks of North America', Wildlife Society Bulletin 26: 449-62.

Whyte, I. J. (2001) 'Headaches and heartaches—the elephant management dilemma', in D. Schmidtz et E. Willot (eds) Environmental Ethics: Introductory readings, pp. 293-305, Oxford University Press, New York.

Wieczorek, J., Bloom, D., Guralnick, R., Blum, S., Döring, M., de Giovanni, R., Robertson, T. et Vieglais, D. (2012) 'Darwin Core: an evolving community-developed biodiversity data standard', PLoS ONE 7(1): e29715. <doi:10.1371/journal. pone.0029715>

Wildlife Conservation Society (WCS) (2002) 'Using conceptual models to set conservation priorities', Living Landscapes Bulletin 5: 1-4.

Williams, W. T. (1980) 'Air pollution disease in the Californian forests: a base line for smog disease on ponderosa and Jeffery pines in the Sequoia and Los Padres National Forests, California', Environmental Science \& Technology 14: 179-82.

Woodley, S. (1993) 'Monitoring and measuring ecosystem integrity in Canadian national parks', in S. J. Woodley, G. Francis et J. Kay (eds) Ecosystem Integrity and the Management of Ecosystems, pp. 155-76, St Lucie Press, Delray Beach, FL.

Dd Woodley, S. (2010) 'Ecological integrity: a framework for ecosystem-based management', in D. N. Cole et L. Yung (eds) Beyond Naturalness: Rethinking park and wilderness stewardship in an era of rapid change, pp. 106-25, Island Press, Washington, DC.

Woodley, S., Bertzky, B., Crawhall, N., Dudley, N., Londońo, J. M., MacKinnon, K., Redford, K. et Sandwith, T. (2012) 'Meeting Aichi Target 11: what does success look like for protected area systems?', PARKS 18(1): 23-36.
Woodley, S., Francis, G. et Kay, J. (eds) (1993) Ecosystem Integrity and the Management of Ecosystems, St Lucie Press, Boca Raton, FL.

Woodwell, G. E. (1970) 'Effects of pollution on the structure and physiology of ecosystems', Ambio 11: $143-8$.

World Checklist of Selected Plant Families (WCSP) (2008) World Checklist of Selected Plant Families, The Board of Trustees of the Royal Botanic Gardens, Kew, Royaume-Uni. <www.kew.org/wcsp>

World Resources Institute (2005) Ecosystems and Human Well-Being: Biodiversity synthesis, World Resources Institute, Washington, DC. 
Ce texte est extrait du livre "Gouvernance et gestion des aires protégées " édité par Graeme L. Worboys, Michael Lockwood, Ashish Kothari, Sue Feary et Ian Pulsford, publié en 2020 par l'ANU Press, l'Australian National University, Canberra, Australie.

La reproduction de cette publication de l'ANU Press à des fins éducatives ou non commerciales est autorisée sans l'autorisation écrite préalable du détenteur des droits d'auteur, à condition que la source soit clairement indiquée. La reproduction de cette publication pour la revente ou à d'autres fins commerciales est interdite sans l'autorisation écrite préalable du détenteur des droits d'auteur. 